\title{
Low Cost Digital Electronics For Isotope Analysis With Microcalorimeters
}

\author{
Final Phase I Report, DOE SBIR No. DE-FG02-05ER84343
}

\section{Execution Summary}

The overall goal of the Phase I research was to demonstrate that the digital readout electronics and filter algorithms developed by XIA for use with HPGe detectors can be adapted to high precision, cryogenic gamma detectors (microcalorimeters) and not only match the current state of the art in terms of energy resolution, but do so at a significantly reduced cost. This would make it economically feasible to instrument large arrays of microcalorimeters and would also allow automation of the setup, calibration and operation of large numbers of channels through software. We expected, and have demonstrated, that this approach would further allow much higher count rates than the optimum filter algorithms currently used.

In particular, in measurements with a microcalorimeter at LLNL, the adapted Pixie-16 spectrometer achieved an energy resolution of $0.062 \%$, significantly better than the targeted resolution of $0.1 \%$ in the Phase I proposal and easily matching resolutions obtained with LLNL readout electronics and optimum filtering $(0.066 \%)$. The theoretical maximum output count rate for the filter settings used to achieve this resolution is about $120 \mathrm{cps}$. If the filter is adjusted for maximum throughput with an energy resolution of $0.1 \%$ or better, rates of $260 \mathrm{cps}$ are possible. This is 20-50 times higher than the maximum count rates of about $5 \mathrm{cps}$ with optimum filters for this detector. While microcalorimeter measurements were limited to count rates of $\sim 1.3 \mathrm{cps}$ due to the strength of available sources, pulser measurements demonstrated that measured energy resolutions were independent of counting rate to output counting rates well in excess of $200 \mathrm{cps}$ or more..

We also developed a preliminary hardware design of a spectrometer module, consisting of a digital processing core and several input options that can be implemented on daughter boards. Depending upon the daughter board, the total parts cost per channel ranged between $\$ 12$ and $\$ 27$, resulting in projected product prices of $\$ 80$ to $\$ 160$ per channel. This demonstrates that a price of $\$ 100$ per channel is economically very feasible for large microcalorimeter arrays. 


\section{Table of Contents}

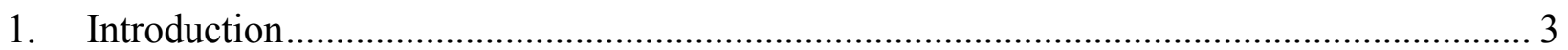

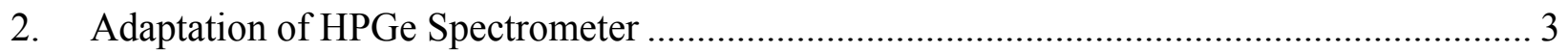

3. Offline Filter Development ................................................................................ 4

3.1. Standard Trapezoidal Filter............................................................................. 4

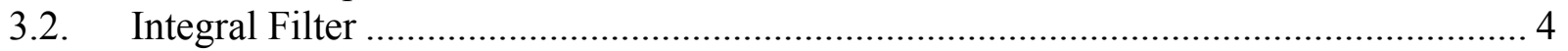

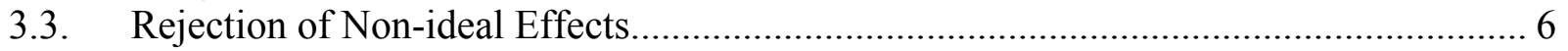

4. Pulser Measurements with Adapted Spectrometer ……................................................... 7

4.1. Measurements with BH-1 Periodic Pulser ................................................................. 7

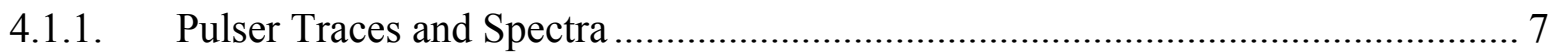

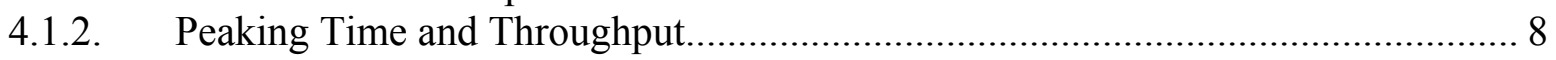

4.1.3. Comparison of Pixie-16 and LLNL Optimum Filters............................................. 9

4.2. Measurements with DB-2 Random Pulser........................................................... 10

4.3. Conclusions from Pulser Measurements.................................................................... 11

5. Microcalorimeter Measurements with Adapted Spectrometer ............................................ 12

5.1. Optimization of Filter Parameters........................................................................ 12

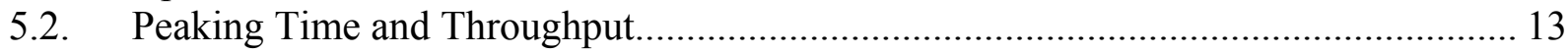

5.3. Comparison to LLNL System ............................................................................... 14

5.4. Conclusions from Microcalorimeter Measurements..................................................... 15

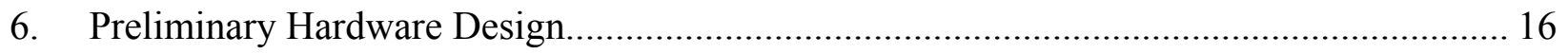

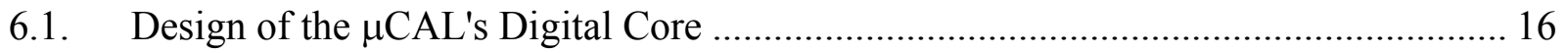

6.2. Daughter Card Input Options ............................................................................. 18

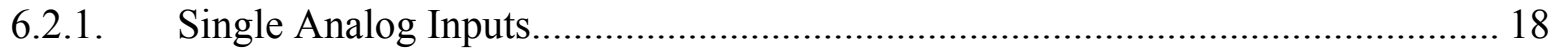

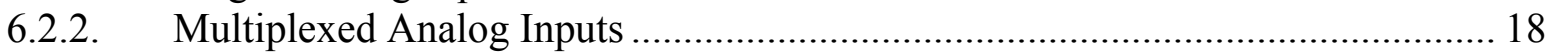

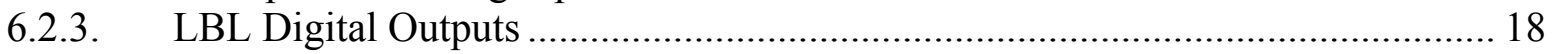

6.3. Hardware Bill of Materials and Pricing ...................................................................... 19

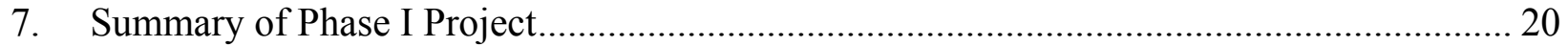




\section{Introduction}

The overall goal of the Phase I research was to demonstrate that the digital readout electronics and filter algorithms developed by XIA for use with HPGe detectors can be adapted to microcalorimeters and not only match the current state of the art in terms of energy resolution, but do so at a significantly reduced cost. This would make it economically feasible to instrument large arrays of microcalorimeters and would also allow automation of the setup, calibration and operation of large numbers of channels through software. We expected, and have demonstrated, that this approach would further allow much higher count rates than the optimum filter algorithms currently used.

Specifically, we had the following objectives for the Phase I efforts:

- Establish that, suitably modified, the hardware architecture and digital filter algorithms used for our HPGe pulse processing electronics would be capable of achieving energy resolutions with microcalorimeters comparable to presently used electronics, which is about $0.1 \%$ at the $122 \mathrm{keV}$ line of $\mathrm{Co}^{57}$.

- By drawing up a preliminary design with a cost estimate for production and components, demonstrate that the electronics could be built at a price of about $\$ 100$ per channel in large quantities.

To achieve these objectives, we carried out the following tasks:

1. Adaptation of HPGe spectrometer to slow microcalorimeter pulses

2. Offline analysis of traces acquired with existing LLNL readout electronics to optimize filter algorithms

3. Pulser measurements to test adapted spectrometer and filters

4. Measurements at LLNL's microcalorimeter

5. Preliminary Hardware design

Through these tasks, which are described in detail in the sections below, we were able to show that we could exceed our energy resolution goal, reaching $0.062 \%$ at the $122 \mathrm{keV}{ }^{57} \mathrm{Co}$ line, could reasonably expect to meet our $\$ 100 /$ channel production target, and, in addition, could process pulses more than an order of magnitude faster than is possible with current methods.

\section{Adaptation of HPGe Spectrometer}

XIA's existing spectrometers for HPGe detectors can acquire pulse waveforms as well as compute pulse heights and bin them in an on-board MCA. However, being designed for pulses with decay time constants in the order of $50 \mu \mathrm{s}$, they sample waveforms as fast as $100 \mathrm{MHz}$ and therefore needed to be adapted to the much slower pulses from microcalorimeters with time constants in the order of 1-10ms or more.

Though we originally proposed adapting a single channel XIA Polaris spectrometer, we decided that it would be better to adapt an XIA Pixie-16 spectrometer instead, since this device has newer and more powerful components and its multi-channel architecture is closer to the final targeted instrument. The only required hardware modification was to adjust the ADC's Nyquist filter to a lower bandwidth, removing noise contributions from frequency ranges irrelevant for the slow pulses. The ADC continued to sample the detector signal at $100 \mathrm{MHz}$, but we inserted an additional circuit at the front end of our Field Programmable Gate Array (FPGA) digital processing firmware to produce block sums of up to $2^{8}$ samples from the digital data stream coming from the ADC. This effectively reduced the sampling interval by the same factor of up to $2^{8}$, or from $10 \mathrm{~ns}$ up to $2.56 \mu \mathrm{s}$. The range of filter time constants implemented in the FPGA was increased by the same factor, which allowed the acquisition of waveforms up to $10 \mathrm{~ms}$ ( $4 \mathrm{~K}$ samples) and a standard trapezoidal filter peaking time of up to $5 \mathrm{~ms}$. While not capable of acquiring a full pulse from microcalorimeters with the longest time constants available at LLNL (e.g. those used for neutrons), this was sufficient to explore the filter parameter space and find optimum settings for the microcalorimeters used for Gamma-ray spectroscopy, eventually reaching an energy resolution matching existing readout methods using optimium filters (see section 5). 


\section{Offline Filter Development}

\subsection{Standard Trapezoidal Filter}

We used offline data from the existing microcalorimeter acquisition system at LLNL to evaluate the various HPGe digital filters, adapt filter parameters, and explore new algorithms. A typical trace from a gamma detector is shown in Figure 1. The standard trapezoidal filter described in section Error! Reference source not found. will first compute the sums over the intervals indicated by black diamonds. The trigger point $T$ is determined by a trigger filter (a simple difference filter whose output is shown in blue) crossing a user defined threshold value,. $\mathrm{E}_{0}$ is the sum over an interval $T_{\text {peak }}$ before the trigger point. $\mathrm{E}_{\mathrm{g}}$ is the sum over an interval $T_{\text {gap }}$ after the trigger point, and $\mathrm{E}_{1}$ is the sum over an interval $T_{\text {peak }}$ following $\mathrm{E}_{\mathrm{g}}$. The energy $\mathrm{E}$ is then computed as

$$
\mathrm{E}=\mathrm{c}_{1} * \mathrm{E}_{1}-\mathrm{c}_{0} * \mathrm{E}_{0}+\mathrm{c}_{\mathrm{g}} * \mathrm{E}_{\mathrm{g}}-<\mathrm{BL}>
$$

where $<\mathrm{BL}>$ stands for contributions of the baseline measurements acquired before the pulse. The coefficients $\mathrm{c}_{\mathrm{i}}$ correct for both the decay of the measured pulse and any underlying decay from a previous pulse and thus depend on the pulse's decay time constant $\tau$.

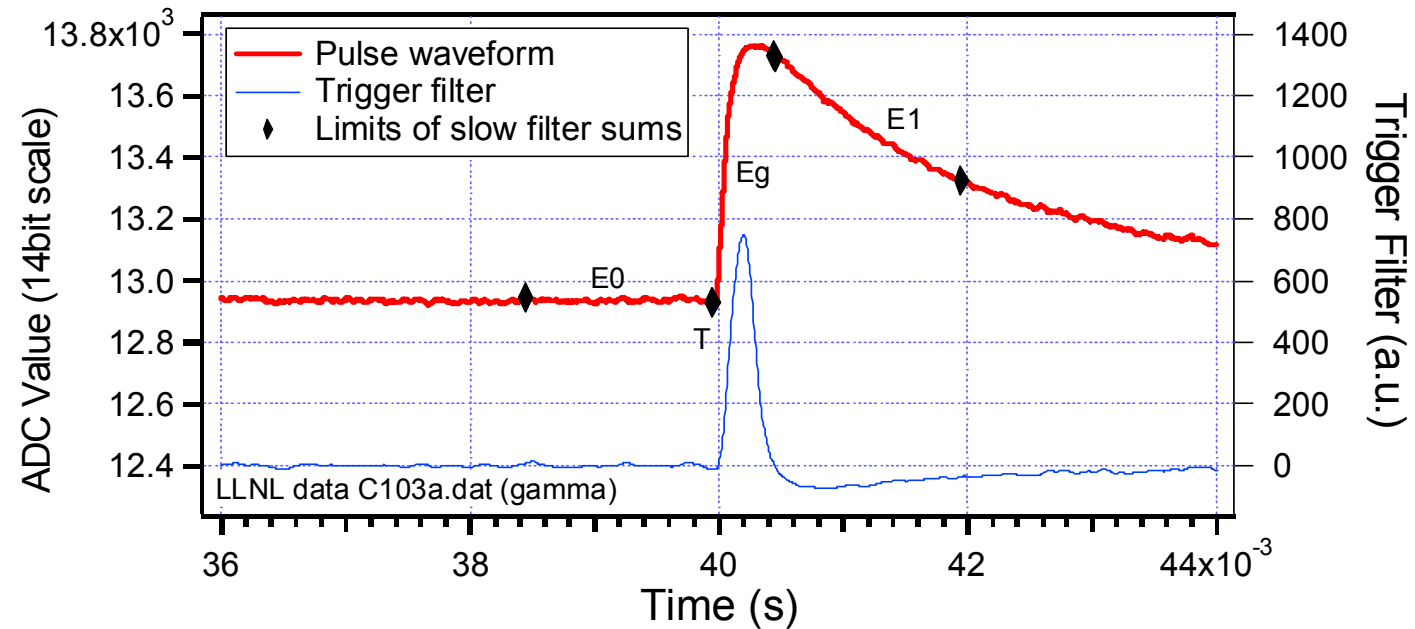

Figure 1: Typical pulse from a gamma microcalorimeter at LLNL (red). The blue line indicates the response of the trigger filter, issuing a trigger when the threshold at 200 is crossed. The black diamonds mark the limits of the energy filter sums.

To get best resolution with this filter, it is important to obtain a correct value of $\tau$, especially if count rates are so high that pulses begin to overlap often. Secondly, the gap time $T_{\text {gap }}$ should cover the rising part of the pulse. For HPGe detectors, this accommodates the arbitrary rising edges created in the detector. For microcalorimeters, since the rising edge can be described by an exponential of the form 1- $\mathrm{e}^{-t / \tau}, T_{\text {gap }}$ should be long enough to fully cover the settling time $\mathrm{e}^{-\mathrm{t} t \mathrm{t}}$. When setting up with a detector, one typically scans through $\tau$ and $T_{\text {gap }}$ to find the values giving the best energy resolution. These values then stay fixed for all further measurements with this detector.

The peaking time $\mathrm{T}_{\text {peak }}$, on the other hand, can be adjusted to find the best tradeoff between energy resolution and throughput. Typically there is an optimum peaking time giving best resolution, but the throughput decreases with longer filter times. This is because the dead time TD associated with the filter is approximately $\mathrm{TD}=2 *\left(\mathrm{~T}_{\mathrm{gap}}+\right.$ $\mathrm{T}_{\text {peak }}$ ), and according to Poisson statistics for paralyzable systems [12], the output count rate (OCR) at a given input count rate (ICR) is

$$
O C R=I C R \exp (-I C R * T D),
$$

which has a maximum $\mathrm{OCR}_{\max }=\left(\mathrm{e}^{*} \mathrm{TD}\right)^{-1}$ at an input count rate of $\mathrm{ICR}_{\max }=(\mathrm{TD})^{-1}$.

\subsection{Integral Filter}

Since real microcalorimeter pulses often show more than one time constant in their decay, a trapezoidal filter assuming a single exponential decay might not give the best energy resolution, especially at higher count rates when pulses often overlap. For pulses with double exponential decays, XIA previously developed a 5 -sum filter achieving 
good energy resolution at high count rate with CsI [13]. However, for the 3 or more decay constants sometimes observed in microcalorimeter pulses at LLNL, which may also include undershoots, 7-sum or 9-sum filters would be required, which makes the algorithms very complex.

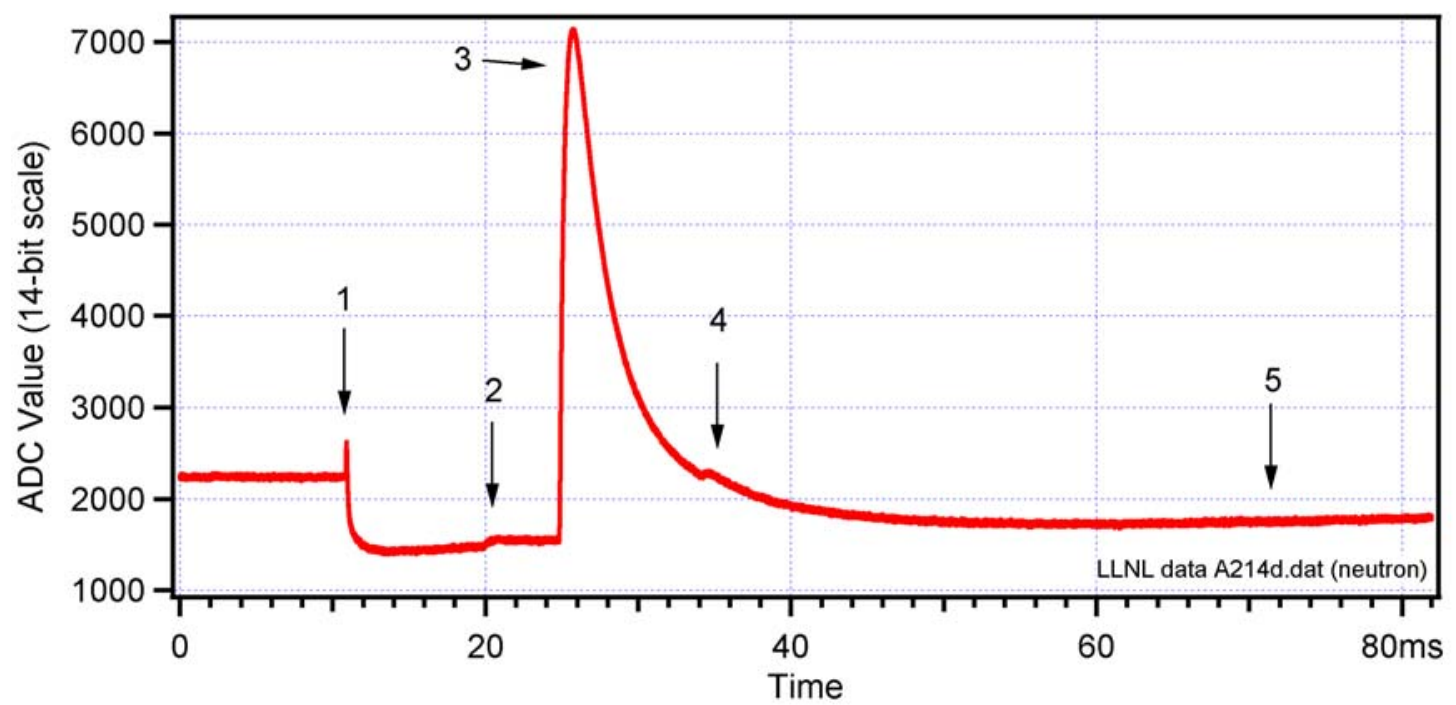

Figure 2: Microcalorimeter waveform (from a slower neutron detector) with non-ideal effects: 1) a fast rising pulse triggering a baseline jump (likely TES was hit instead of absorber); 2) baseline jump (note no decay); 3) main pulse with double exponential decay; 4) a second, weak overlapping pulse; and 5) an overall undershoot.

An example of a waveform from one particular (neutron) detector with a large number of decays and/or undershoots is shown in Figure 2. Note that, from the spacing of the events $2-4$, both the main pulse at (3) and the second pulse at (4) can be processed and their pulse height measured using a filter having a peaking time of up to $3 \mathrm{~ms}$, which was found to be more than adequate for good energy resolution with isolated pulses. We therefore developed a new integral filter (Figure 3) that approximates the pretrigger baseline with a single exponential decay or undershoot, extrapolates this pretrigger time to the pulse area, and then calculates the energy as the difference between the integral over the pulse and the integral over the extrapolated baseline. In designing this filter, we made use of the fact that the rising and falling parts of the pulses from a given microcalorimeter always have the same shape, so that an integral over a fixed portion of the pulse is proportional to the pulse height. The algorithm assumes that the majority of pulses are sufficiently separated so that there is only one major exponential left, but allows for the different decay constants or undershoots, i.e. the algorithm tolerates cases where the pretrigger decay may be different from the decay of the pulse to be measured. At low count rates with mostly flat pretrigger baselines, an integral over the measured pretrigger baseline can be substituted for the integral over the extrapolated baseline.

This method has the advantage that it does not need a predetermined value of the decay time(s), and that it can process pulses that would otherwise have to be rejected completely. For example, it can handle the different pretrigger behaviors for pulses (3) and (4) as well as any possible additional pulses between (1) and (2) or at (5), i.e. the cases of flat baseline, slow exponential decay, fast exponential decay, and undershoot. While the offline analysis used a standard fit routine to determine the baseline, online calculations can perform the equivalent operations: offset, amplitude and decay constant of the baseline can be determined from appropriate sets of filter sums and a value corresponding to the integral over the extrapolated baseline can be calculated from these parameters. 


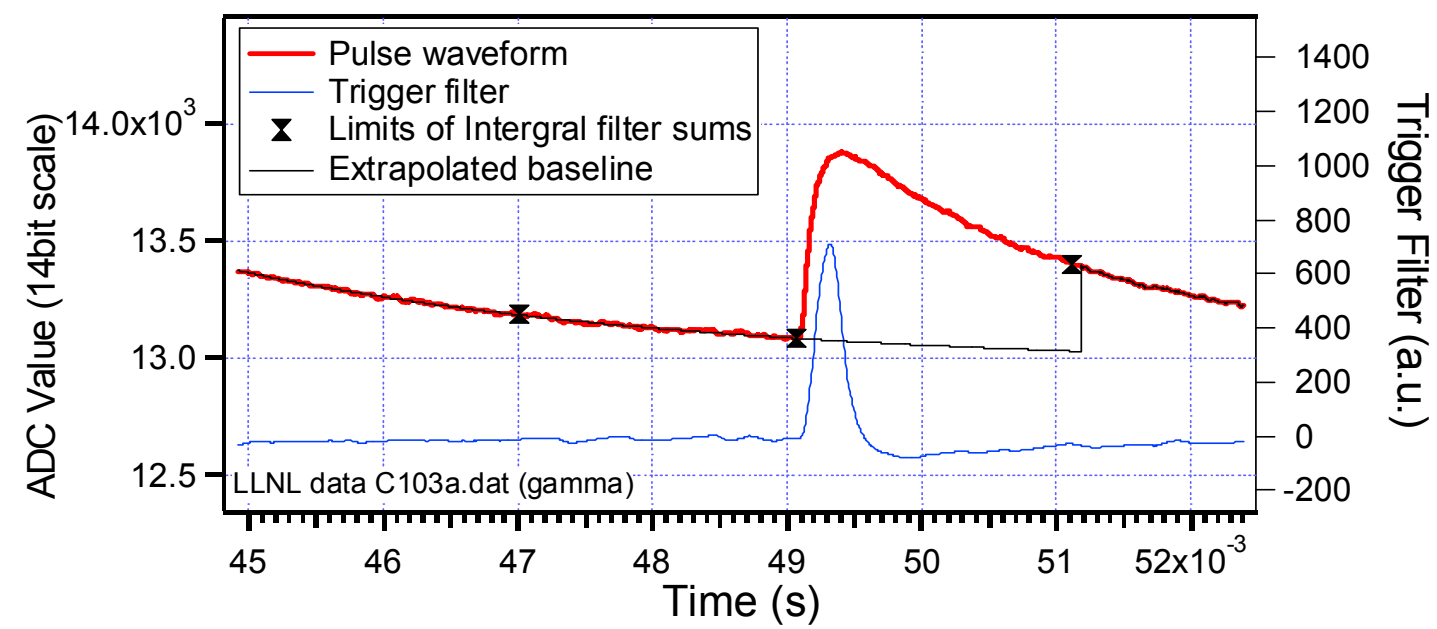

Figure 3: Integral filter applied to microcalorimeter pulse (from a comparatively fast gamma detector) with decay from previous pulse. The pulse height is determined from the difference of the integral over a portion of the pulse and an equivalent integral over an extrapolated baseline.

The integral filter is most suitable for detectors with high occurrence of the non-ideal effects. Detectors with well defined double exponential decays will still obtain best results with the previously developed 5-sum filter. Since for the detectors used in Phase I the standard slow filter and the integral filter both gave good results, we have deferred further investigation of the 5-sum filter to Phase II of the project.

\subsection{Rejection of Non-ideal Effects}

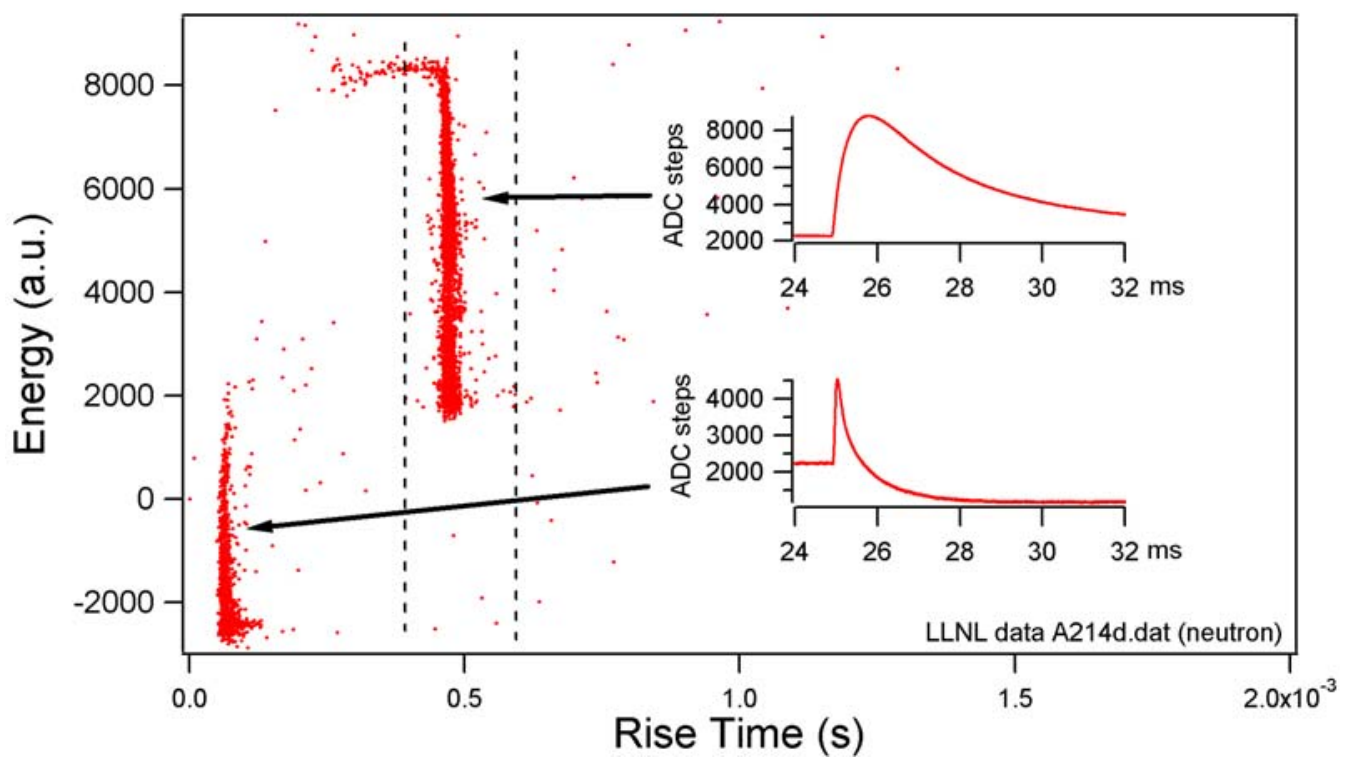

Figure 4: Risetime cut for fast rising pulses.

The waveform shown in Figure 2 also show two further non-ideal effects besides multiple decay constants: Fast rising pulses due to Gamma-ray hits in the TES rather than the absorber (1) and baseline jumps that are sometimes triggered by such TES hits $(1,2)$. The fast rising pulses can be rejected from the acquired data by calculating the risetime of each pulse, and then defining rejection cuts to only accept those events with a risetime corresponding to proper hits.

Figure 4 shows a scatter plot of energy vs risetime of a large number of events. One can see that the events fall into two groups with different risetimes, one corresponding to slow rising normal pulses, the other to fast rising 
pulses. A risetime cut can be implemented to reject the fast rising pulses. This can be done offline or with the onboard firmware and software of our proposed readout electronics.

\section{Pulser Measurements with Adapted Spectrometer}

The adapted Pixie-16 hardware and software were then tested with a number of pulsers at XIA as in-house substitutes for microcalorimeters. This work had several goals. The first was to determine that our processing algorithms were working correctly. Secondly we wanted to investigate a) the extent to which energy resolution might be limited by our hardware (number of ADC bits, input noise, etc.); and b) how well energy resolution held up at high ICR values. Since the pulsers show moderately well behaved single exponential decays even at high count rates, most of these measurements were done using on-line energy calculations with our standard trapezoidal filter, i.e. only the spectra were read out to the host computer. A limited number of waveforms was acquired for verification of the offline algorithms.

\subsection{Measurements with BH-1 Periodic Pulser}

\subsubsection{Pulser Traces and Spectra}

A Berkeley Nucleonics Pulser (Model BH-1) was set to generate pulses with a rise time of $50 \mu$ s and a fall time of $1 \mathrm{~ms}$, which are similar to the typical pulse characteristics of a gamma microcalorimeter (rise time 10-100 $\mu$ s, fall time $1-10 \mathrm{~ms}$ ). However, while the pulses generated by the BH-1 initially have a precise exponential decay, they cut off sharply before they are fully decayed (Figure 5). This is not a problem for low count rates as only the initial part of the pulse is used for the energy calculation with the trapezoidal filters, but may affect baseline measurements at higher count rates when the cut off is close to a following pulse.

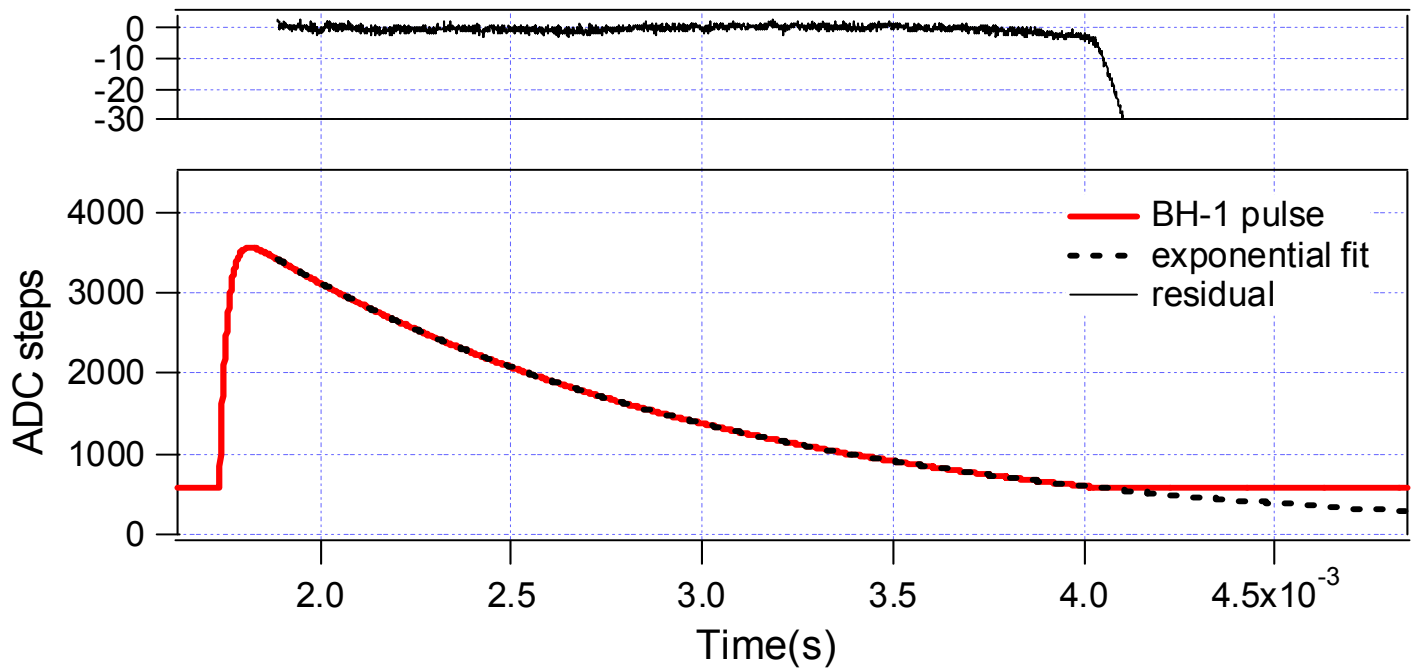

Figure 5: Pulse from BH-1 periodic pulser and exponential fit to the initial pulse tail. Note the sharp cut-off at about $4 \mathrm{~ms}$.

Parameters for the slowed digital filters implemented in the Pixie-16 were optimized according to the pulser characteristics. A decay time of $1.1 \mathrm{~ms}$ and a gap time of 120 to $160 \mu$ s were found to give the best results. Settings of the peaking time affect the maximum throughput and are discussed in the next section. 


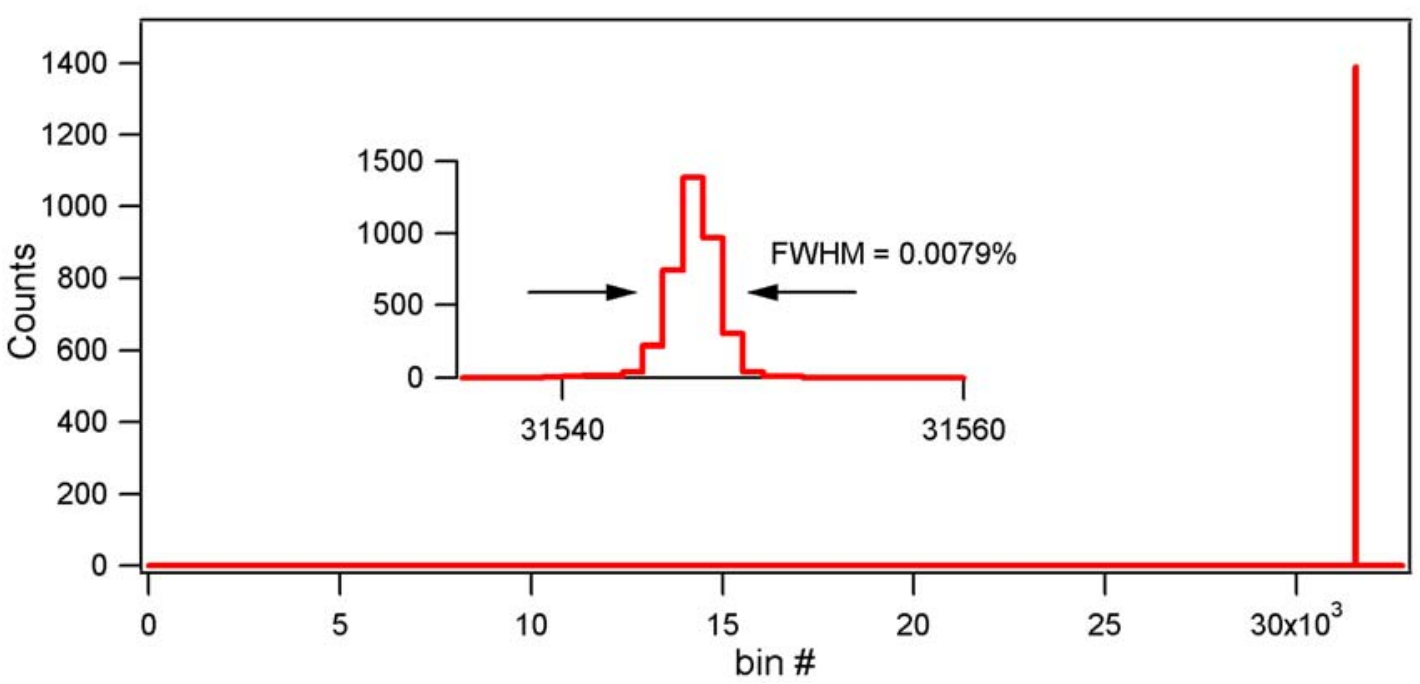

Figure 6: MCA spectrum from BH-1 pulser with optimized amplitude, gain and attenuation of the pulser.

While the resolutions measured within an acquisition session are consistent and repeatable, the energy resolutions obtained from this pulser depend substantially on both the pulser settings and signal conditioning. It appears that the BH-1 pulser is built such that a constant noise is added to a variable amplitude. The signal to noise ratio thus varies with the pulse amplitude; larger signals giving better energy resolution than smaller signals. In addition, the pulser has attenuation options that further affect the noise. For practical reasons we set the pulser amplitude at about 3000ADC steps of the Pixie16 (out of 4096 maximum) without attenuation and obtained energy resolutions in the range of $0.02-0.05 \%$. In a complex and highly optimized setting (using larger pulses and adjusting the pulser's gain and attenuation), the Pixie-16 even achieved a resolution of $0.0079 \%$ (see Figure 6). All of these values are significantly better than our target goal of $0.1 \%$, so the Pixie hardware will not be a limiting factor.

\subsubsection{Peaking Time and Throughput}

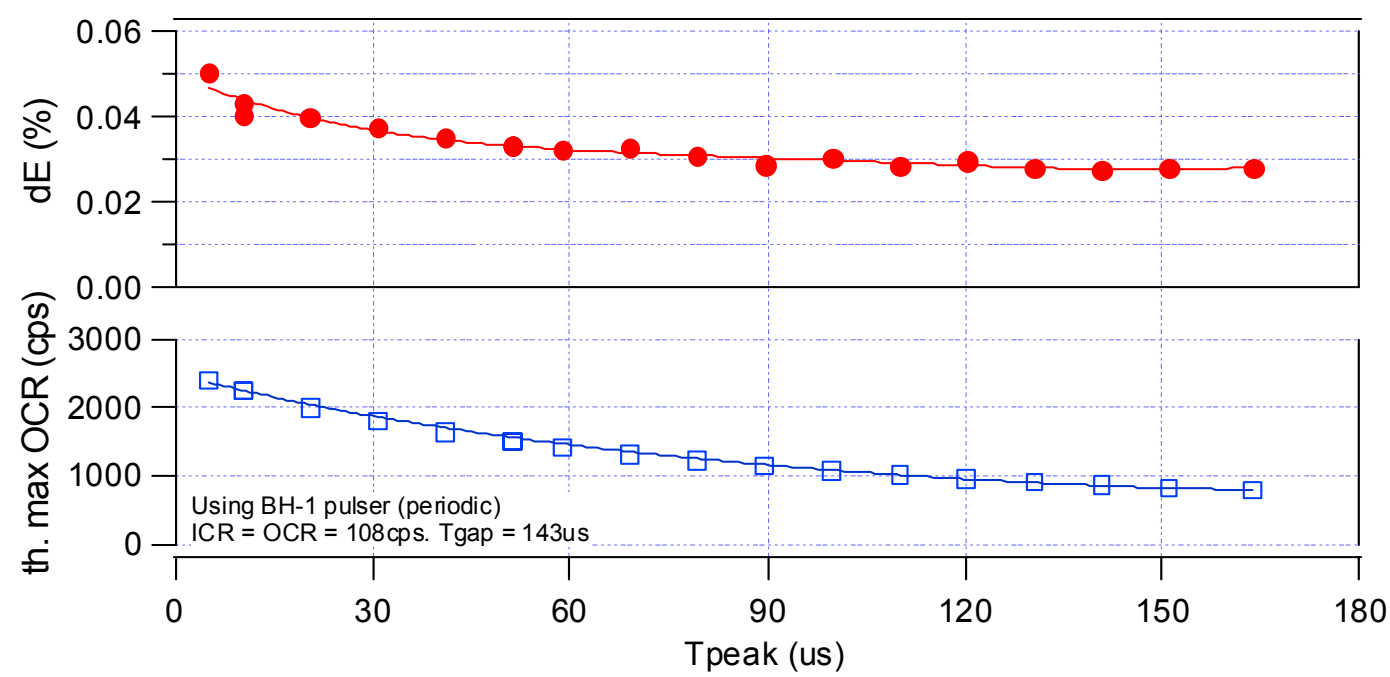

Figure 7. Resolution and maximum theoretical throughput (assuming Poisson statistics) as a function of peaking time. The measurement at $51.2 \mu$ s was repeated 3 times to give a rough idea of the error bars.

To optimize the peaking time (usually the most significant parameter affecting the throughput), decay time and gap time were fixed and the peaking time was varied from $5.12 \mu$ s to $179.2 \mu \mathrm{s}$. The results are shown in Figure 7. For peaking times above $90 \mu \mathrm{s}$, the resolution is below $0.03 \%$. If the peaking time is reduced to $30 \mu \mathrm{s}$, the resolution increases to $0.04 \%$, and eventually to $0.05 \%$ for the smallest peaking time $(5.1 \mu \mathrm{s})$. These curves essentially 
"characterize" the noise characteristics of the pulser, identifying both the peaking time that provides the best energy resolution and also quantifying the tradeoffs between energy resolution and throughput at shorter peaking times.

From the data we can also compute the maximum theoretical throughput (OCR) for each filter time, assuming Poisson statistics for incoming pulses according to Eqn. 3 (above). At longer peaking times, the filter dead time is increased and the maximum throughput is reduced. However, even for the longest peaking time the maximum throughput is above 500cps. For best accuracy of spectrum analysis, a compromise between high throughput and good resolution has to be found (ref. section Error! Reference source not found.), which will vary according to the applications. For our further measurements, we used a peaking time of $51.2 \mu \mathrm{s}$, which gives a maximum theoretical throughput of about $1600 \mathrm{cps}$.

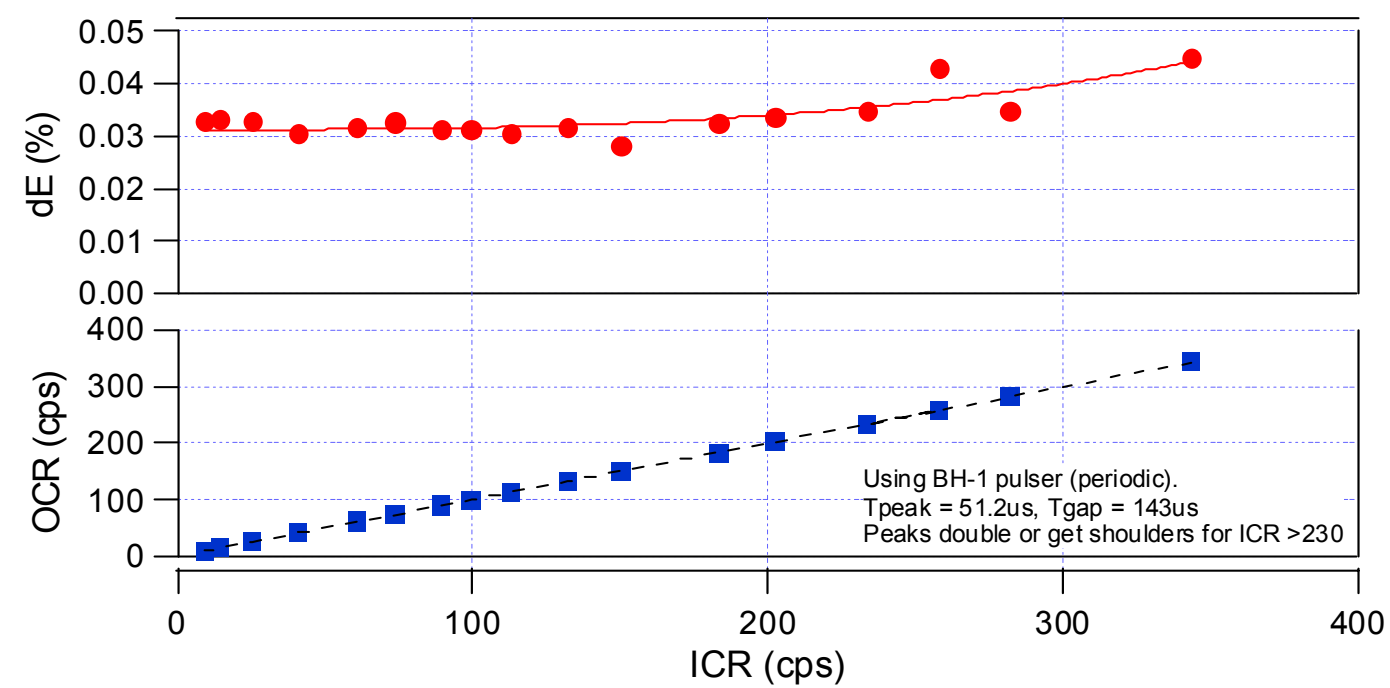

Figure 8. Resolution and measured throughput as a function of ICR. The black doted line is the expected relation OCR $=\mathbf{I C R}$

While the filter time limits the maximum throughput of the digital filter algorithms, resolution at a given filter time typically depends on the actual count rate, since other factors such as baseline correction come into play. Therefore, using the compromise value of the peaking time determined above $(51.2 \mu \mathrm{s})$, we varied the pulser rate and measured the actual achieved output count rate and energy resolution as a function of input count rate. Of course, OCR = ICR for a periodic pulser which does not follow Poisson statistics until the pulses become too close together to be processed at all.

We found that the resolution remains at about $0.03 \%$ from lowest count rates up to about $230 \mathrm{cps}$ (see Figure 8), at which point the peaks in the spectrum distort and develop shoulders and double peaks. We attribute this behavior to the non-ideal output of the pulser, especially the sudden cut off of the pulse. When this cut-off falls into the baseline measurements of the digital filter, it results in different baseline values for the decay and the flat region. Nevertheless, the count rate is much higher than what could be achieved with optimum filtering, and the resolution always remains well below the $0.1 \%$ value targeted for microcalorimeters.

\subsubsection{Comparison of Pixie-16 and LLNL Optimum Filters}

The same BH-1 pulser was taken to LLNL during measurements with the gamma microcalorimeter. and spectra were taken with both the LLNL acquisition system and the adapted Pixie-16. At a count rate of 134cps, using a peaking time of $158.71 \mu$ s and a gap time of $120.32 \mu$ s, the Pixie- 16 reached a resolution of $0.029 \%$. The LLNL system reached $0.291 \%$ without the optimum filtering applied and $0.088 \%$ with optimum filtering (Figure 9). The Pixie-16 thus achieved better energy resolution from the pulser signals than the optimum filter algorithms (though admittedly there was not too much time to optimize setup and parameters of the LLNL system). 

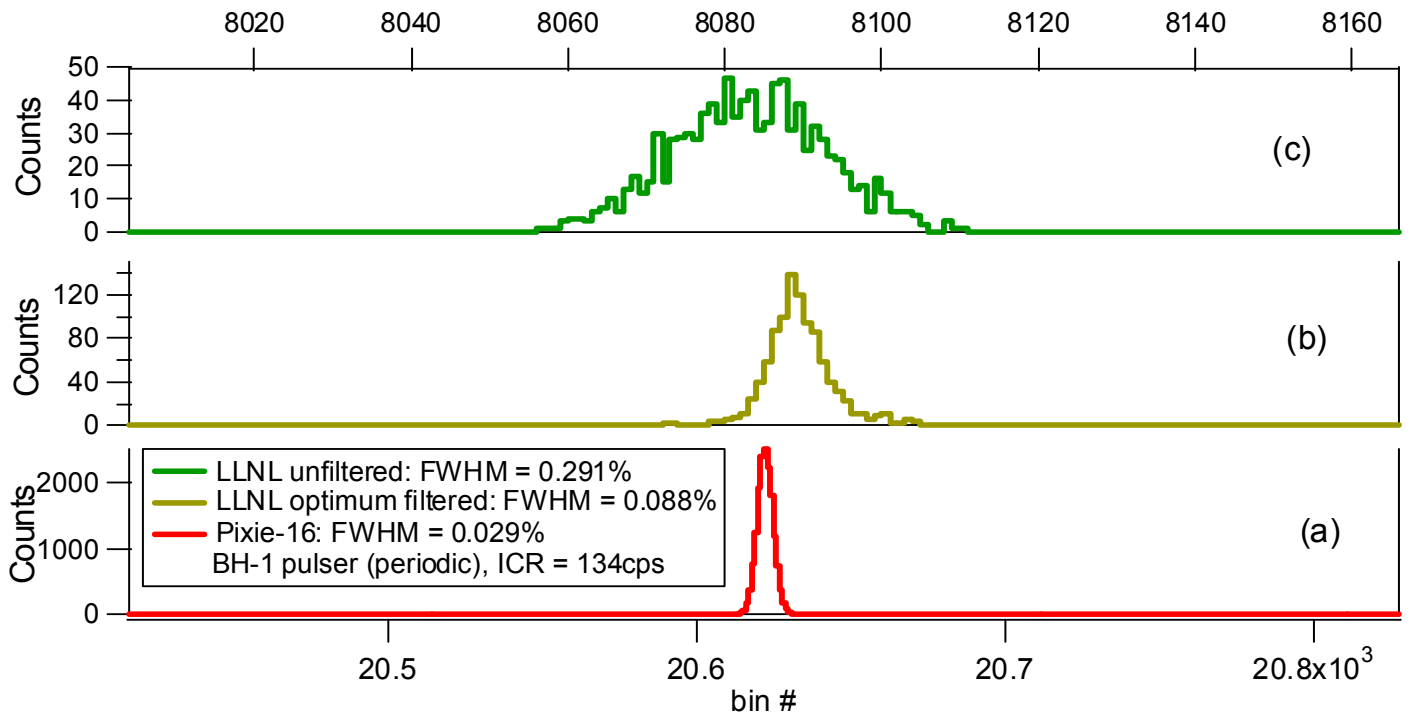

Figure 9. MCA spectra of BH-1 pulser, acquired with Pixie-16 (a) and LLNL system with (b) and without (c) optimum filtering. ThePixie-16 was set to generate a 32K spectrum, the LLNL system generated 8K spectra; the plot is adjusted to equal binning scale. The pulse height was the same in both measurements.

\subsection{Measurements with DB-2 Random Pulser}

Since pulses from the BH-1 periodic pulser do not properly overlap at higher count rates (but true microcalorimeter pulses will), further measurements were performed with a Berkeley Nucleonics DB-2 random pulser, which can also generate signals in periodic mode. The Pixie-16 settings were again optimized according to the pulser signal, which had a nominal rise time of $50 \mu$ s and a fall time of $1 \mathrm{~ms}$. The DB-2 pulser did not cut off the pulses' exponential tails abruptly, as the BH-1 did, however it showed a pronounced undershoot at the end of the pulses.

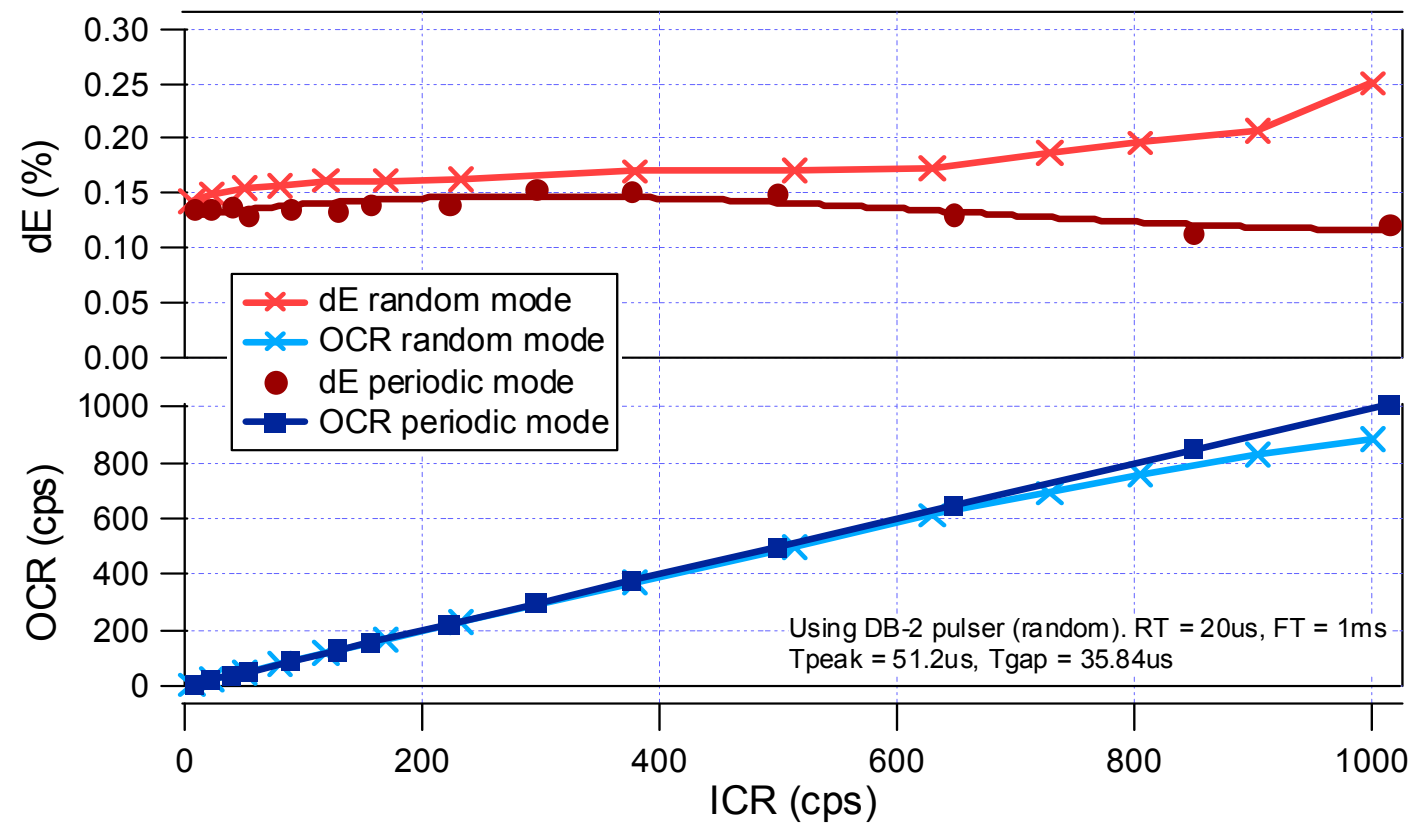

Figure 10: Energy resolution and output count rate from DB-2 random pulser in random and periodic mode. OCR=ICR in periodic mode, and only slightly below ICR in random mode. 
For the DB-2 pulser, the energy resolution depends on the amplitude of the pulse, similarly to the BH-1 pulser, since the output noise is independent of the amplitude. This resulted in degraded energy resolution in these experiments since, at high count rates in random mode, the pulse amplitude must be set to not more than about $1 / 3$ of the ADC's available range so that even when several pulses happen to follow each other very closely, their combined amplitude does not go out of range. Consequently, with the signal reduced by -3 , the DB-2's signal to noise ratio in these measurements was more than a factor of three worse than the BH-1's and certainly less than optimum.

At these pulser settings, the energy resolution both in random and periodic mode is about $0.15 \%$ at low count rates (Figure 10). In periodic mode, the resolution remained essentially constant even at rates up to $1000 \mathrm{cps}$. In random mode, it increased only slightly up to $\sim 600 \mathrm{cps}(0.17 \%)$ and remained below $0.2 \%$ up to $\sim 800 \mathrm{cps}$, but worsened markedly above that. We attribute this behavior to the undershoot of the signal. At high count rates, the pulses randomly overlap with either the decay or the undershoot of a previous pulse while the filter algorithms can account only for the decay. In periodic mode, the contribution of the previous pulse is always the same.

Note that even in random mode when pulses overlap frequently, OCR is very close to ICR. For example, even at an input count rate of $630 \mathrm{cps}$ (see Figure 11), 97\% of all incoming pulses are processed into the spectrum. This is because, for the peaking time $(51.2 \mu \mathrm{s})$ and gap time $(35.84 \mu \mathrm{s})$ used, the dead time is only $0.174 \mathrm{~ms}$ and $\mathrm{OCR}_{\max }$ is over $2100 \mathrm{cps}$. As long as pulses are separated by at least $87 \mu$ s they can be processed. These pulser experiments demonstrate the ability of the modified processor to operate at high throughputs and to maintain energy resolution even when consecutive pulses partially overlap one another.

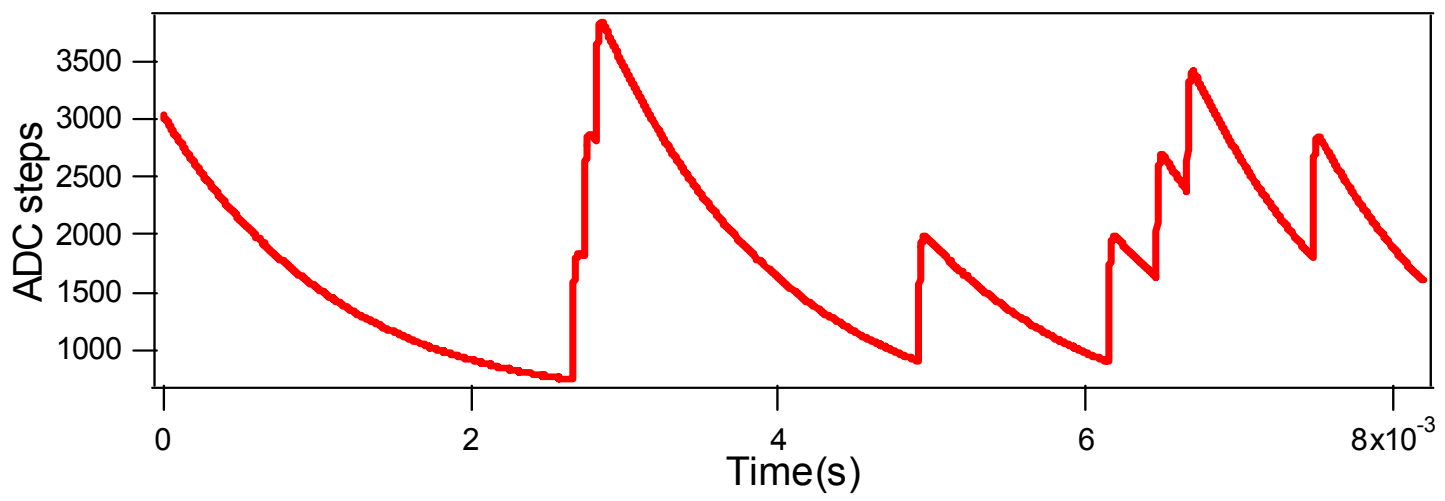

Figure 11: DB-2 random pulser signal at $630 \mathrm{cps}$. Note that only the three pulses at about $\mathrm{t}=2.6 \mathrm{~ms}$ can not be processed due to pulse pileup, all others are sufficiently separated for energy measurements with the trapezoidal filters.

\subsection{Conclusions from Pulser Measurements}

The pulser measurements demonstrated that the adapted hardware and digital filter algorithms worked very well with pulser signal having similar time constants to microcalorimeters. For periodic pulses from the BH-1 pulser, the achieved resolutions of $\sim 0.030 \%$ are well below the $0.1 \%$ targeted for microcalorimeters. These resolutions were measured for count rates up to $200 \mathrm{cps}$, and could in theory be achieved for rates up to $\sim 1600 \mathrm{cps}$ except for imperfections of the signal source. For random pulses from the DB-2 pulser, resolutions are between $0.14 \%$ and $0.20 \%$ due to the required lower pulse amplitudes and thus higher relative noise contributions from the pulser. However, measured resolutions do not degrade significantly up to count rates of $600 \mathrm{cps}$.

In contrast, optimum filters would require pulses to be separated by at least $5 \mathrm{~ms}$ (see Figure 5) in order to capture complete pulses. This would result in a dead time TD of twice that, or $10 \mathrm{~ms}$. This would limit throughput to $200 \mathrm{cps}$ for periodic signals and following Eqn. 3 for random signals, to a maximum theoretical throughput OCR $_{\max }$ of only about $35 \mathrm{cps}$. This demonstrates, for pulsers at least, a major advantage of our trapezoidal filtering algorithms, namely an increase in throughput of at least a factor of 20 over optimum filtering. In the following sections we will show that the same result also holds for real microcalorimeter pulses.

The measurement clearly emphasize that both the signal source and pulse shape are very important to achieve the best resolution. The pulse characteristics of the BH-1 and DB-2 pulsers were very similar in terms of nominal 
rise and fall times, yet the resolutions measured differed by about a factor two. Subtle differences in amplitude, gain and attenuation as well as effects like undershoots can significantly affect the resolution.

In summary, the pulser measurements showed that our adapted hardware and trapezoidal filters can achieve better than $0.1 \%$ resolution at high count rates for slow pulses, but also stress the fact that the filters require a suitable signal source and/or careful optimization to the characteristics of the signal source to do so.

\section{Microcalorimeter Measurements with Adapted Spectrometer}

\subsection{Optimization of Filter Parameters}
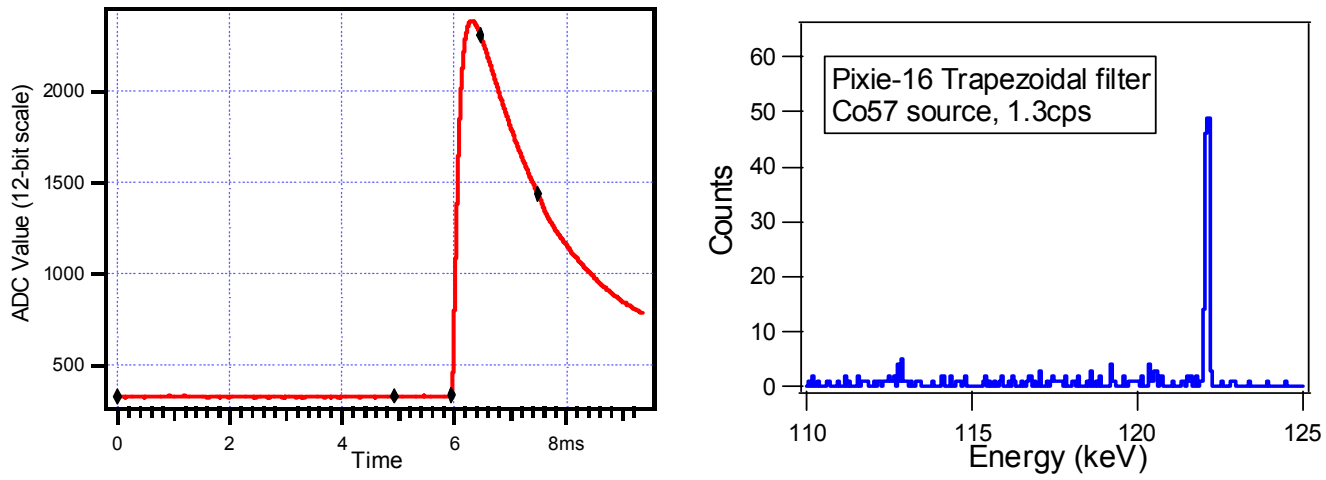

Figure 12: Microcalorimeter trace and typical Co57 spectrum

The adapted Pixie-16 hardware and software were employed at LLNL with a microcalorimeter for gamma spectroscopy in several measurement sessions. The samples available during these sessions limited the count rate to about $1.3 \mathrm{cps}$. Since acquisition time was finite, the Pixie-16 was used to acquire a large number of waveforms which were then processed offline to adjust filter parameters for best energy resolution. While the hardware is fully capable of processing pulses on-line, as shown in the pulser measurements, carefully optimizing the filter parameters by acquiring on-line spectra for many combinations of parameter values would be prohibitively time consuming in the context of our limited LLNL access time. We also noted slight jumps in detector gain over time, which can cause double peaks in on-line spectra that do not detect and correct for this behavior. Processing pulses offline allowed us to correct or reject data from different gain periods. It is worth noting that this is also LLNL's standard procedure with their current processing methods. Obviously an important Phase II task will be to develop alghorithms to detect and correct for these gain shifts on-line.

Figure 12 shows a waveform acquired with the Pixie-16 from microcalorimeter intended for neutron detection. The rise time is about $500 \mu \mathrm{s}$, the decay has two exponential components with about $2.2 \mathrm{~ms}$ and $13.5 \mathrm{~ms}$. This particular detector did not exhibit undershoots and had only a few fast rising pulses. The Pixie-16 acquired waveforms $9.3 \mathrm{~ms}$ in length, which is shorter than a full decay of the pulse, but allowed exploration of a sufficient range of filter parameters. Also shown in Figure 12 is a MCA spectrum from the ${ }^{57}$ Co source.

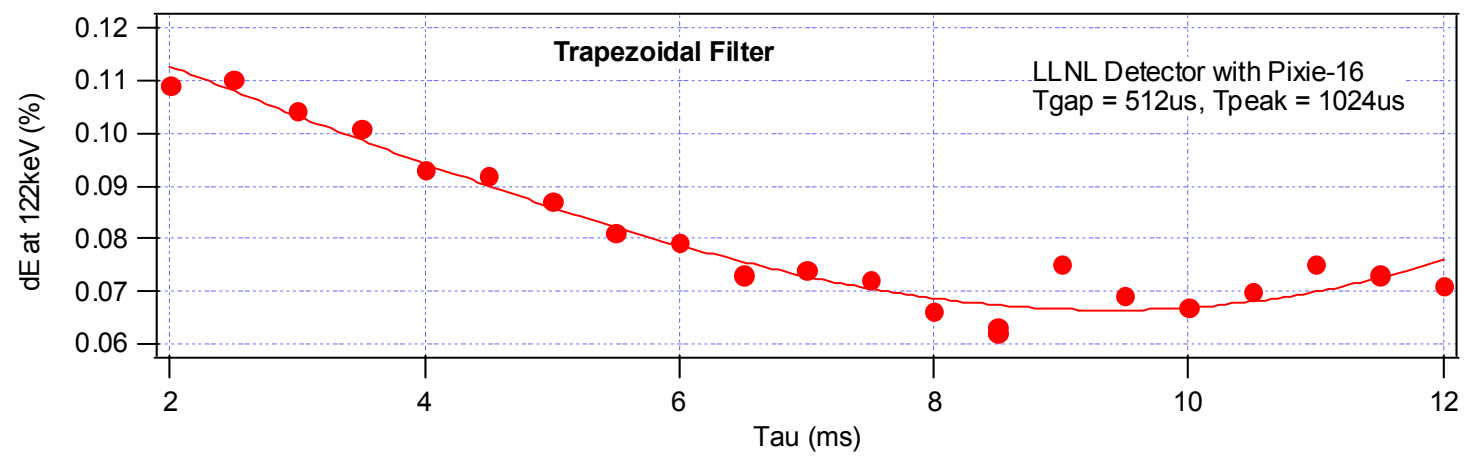


Figure 13: Optimizing the decay constant $\tau$ for the microcalorimeter pulses.

The acquired data was first used to determine the optimum gap time and decay time $\tau$ for the trapezoidal filter. Once optimum values are found, these detector characteristics can be kept constant for most of the subsequent analysis. Using an initial estimate value of peaking time and gap time, $\tau$ was varied from $2 \mathrm{~ms}$ to $12 \mathrm{~ms}$, see Figure 13 . The best energy resolution is obtained at $\tau=8.5 \mathrm{~ms}$, an intermediate value of the two decay constants of the pulse.

With the above value of $\tau$, Tpeak was held constant and Tgap was varied from $250 \mu$ s to $800 \mu$ s, see Figure 14 . The best energy resolution is obtained for Tgap $=512 \mu \mathrm{s}$, a value close to the observed signal rise time.

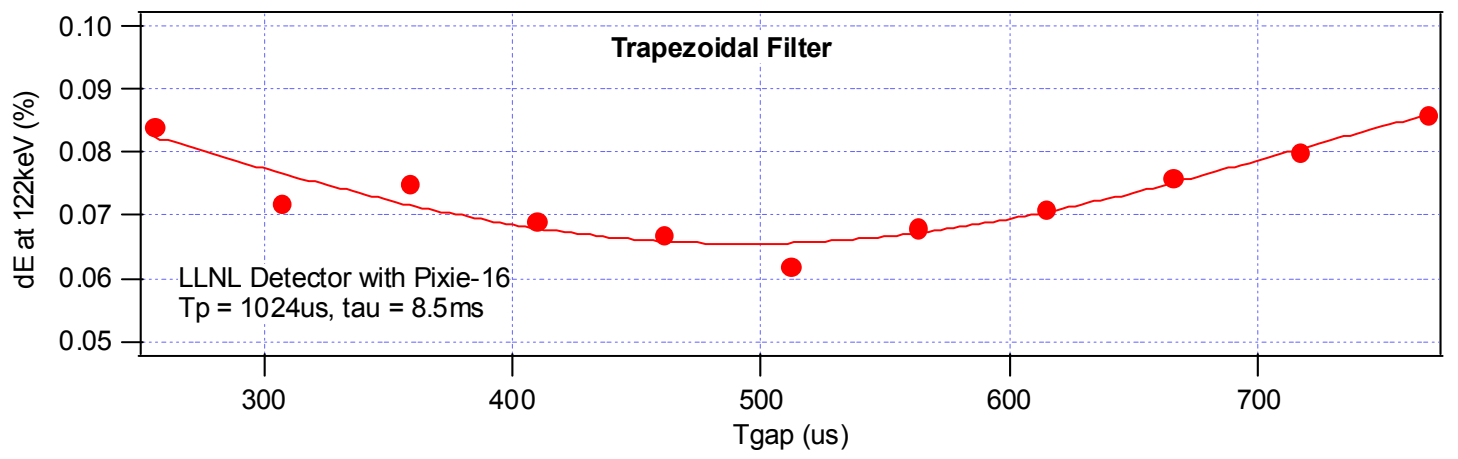

Figure 14: Optimizing the gap time $T_{\text {gap }}$ for the microcalorimeter pulses.

\subsection{Peaking Time and Throughput}

With the above values of $\tau$ and Tgap, Tpeak was varied from $50 \mu$ s to $1700 \mu \mathrm{s}$. The best energy resolution of $0.062 \%(76 \mathrm{eV})$ is obtained for Tpeak $=1024 \mu \mathrm{s}$, see Figure 15 . This resolution is significantly better than the targeted minimum achievement of $0.1 \%$, and at least as good as the resolution obtained with existing LLNL electronics and optimum filtering in the same measurement period (see section 5.3). The dependence of the maximum theoretical throughput as a function of the peaking time is shown in the same figure: For the filter setting that achieves a resolution of $0.062 \%$, the maximum output counting rate $\mathrm{OCR}_{\max }$ is about $120 \mathrm{cps}$. Up to $260 \mathrm{cps}$ can be processed using a peaking time of $200 \mu$ s while still obtaining a resolution better than $0.1 \%$. Both from the pulser experiments reported above and from our years of experience using trapezoidal filters with other detectors, we are fully confident that these throughputs can be achieved with microcalorimeter pulses as well when more intense sources become available.

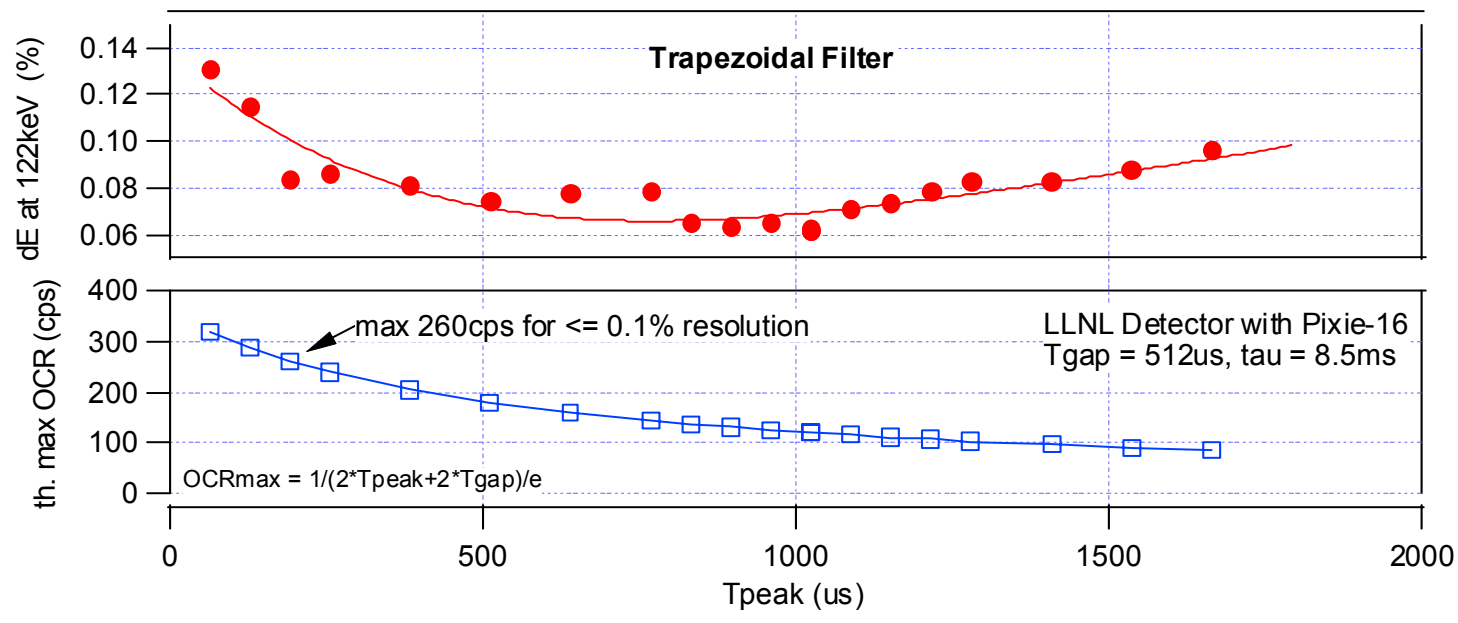

Figure 15: Energy resolution and maximum theoretical throughput as a function of peaking time.

Since this microcalorimeter's pulses are well behaved, the trapezoidal filter gives good results and the flexibility of the integral filter is not required at low count rates. However, at higher count rates, the pulses will 
begin to overlap and the energy resolution with trapezoidal filters may worsen due to the double exponential decay. In contrast, the integral filter will still perform well even at overlapping pulses, since a) in the vast majority of pulses only the $13 \mathrm{~ms}$ decay component will remain from a previous pulse, and b) the filter does not depend on a fixed decay constant for the pulse to be measured. As shown in Figure 16, the integral filter also reaches a good energy resolution of $0.065 \%(79 \mathrm{eV})$, though the count rate for resolutions better than $0.1 \%$ is limited to $100 \mathrm{cps}$.
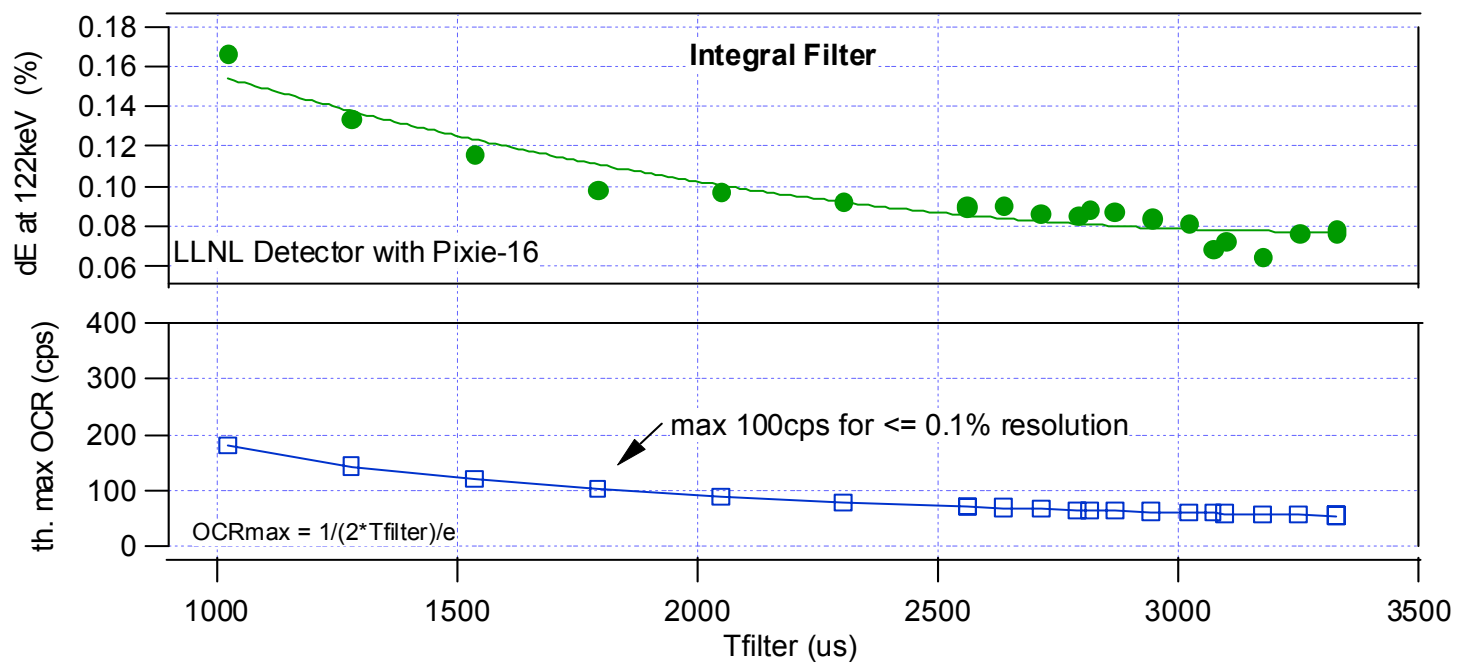

Figure 16: Energy resolution and maximum theoretical throughput as a function of integral time for the integral filter.

\subsection{Comparison to LLNL System}

For a direct comparison between XIA and LLNL's algorithms, we applied the same off-line processing methods described above to waveforms acquired with the existing LLNL system from the same detector in the same time frame. The LLNL waveforms were $80 \mathrm{~ms}$ long compared to the $9.3 \mathrm{~ms}$ acquired with the Pixie- 16 . This allowed the use of much longer filter times and longer baseline averages. A best resolution of $0.062 \%$ (76eV) was found for a peaking time of $\sim 1 \mathrm{~ms}$ and a gap time of $\sim 0.45 \mathrm{~ms}$, which is essentially the same resolution, and for almost the same filter parameters, as for our results from the data acquired with the Pixie-16. At this filter time, the maximum theoretical throughput $\mathrm{OCR}_{\max }$ is $150 \mathrm{cps}$. If the peaking time is reduced to $0.6 \mathrm{~ms}$, the resolution increases to only $0.093 \%$, but the maximum theoretical throughput increases to about $220 \mathrm{cps}$. This shows that our results fundamentally derive from our processing algorithms and not from the details of how the data pulses are sampled and collected.

LLNL processed this same data with optimum filtering and obtained a resolution of about $80 \mathrm{eV}(0.066 \%)$, essentially matching the resolution obtained with the trapezoidal filters. However, since their optimum filter algorithms require $\sim 40 \mathrm{~ms}$ worth of undisturbed waveform for each pulse, their method's dead time TD is $\sim 80 \mathrm{~ms}$, limiting their maximum output count rate $\mathrm{OCR}_{\max }$ to $\left(\mathrm{e}^{*} 0.08 \mathrm{sec}\right)^{-1}$ or less than $5 \mathrm{cps}$, a factor of almost 30 less than our trapezoidal filters' $\mathrm{OCR}_{\max }$. This, of course is a major reason why a stronger source was not available, since LLNL's existing methods would not have been able to deal with it. 


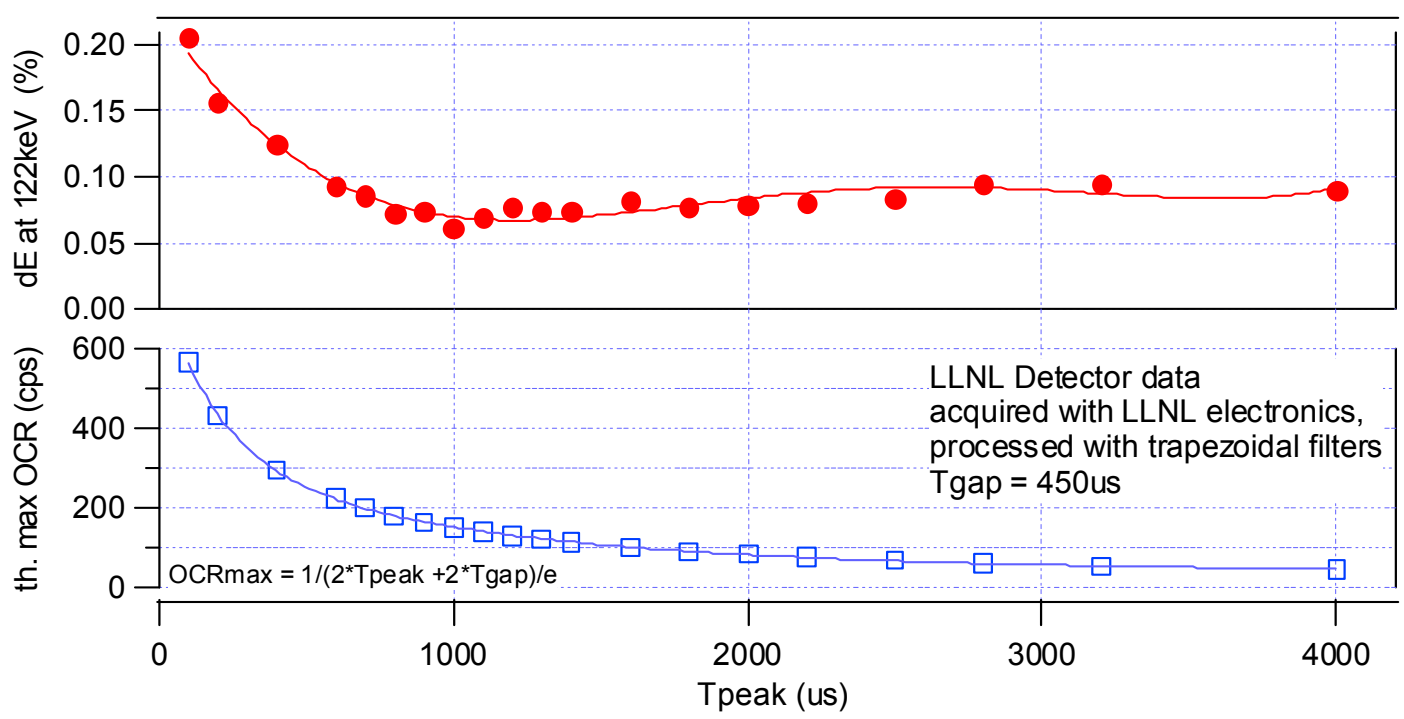

Figure 17: Data acquired by LLNL readout electronics processed with trapezoidal filters.

\subsection{Conclusions from Microcalorimeter Measurements}

In summary:

1. the adapted Pixie- 16 spectrometer achieved an energy resolution of $0.062 \%$, significantly better than the targeted minimum resolution of $0.1 \%$ from the Phase I proposal.

2. The maximum theoretical output count rate from the filters used to achieve this resolution is about $120 \mathrm{cps}$.

3. If the filter is adjusted for maximum throughput with an energy resolution of $0.1 \%$ or better, rates of $260 \mathrm{cps}$ are possible.

4. The resolutions achieved with the Pixie-16 match or slightly surpass the resolutions achieved with LLNL readout electronics and optimum filtering $(0.062 \%$ compared to $0.066 \%)$.

5. Waveform capture using the Pixie-16 is as precise as acquisition with the LLNL system, since the same energy resolution is obtained by applying offline trapezoidal filters both to Pixie-16 waveforms and to LLNL waveforms $(0.062 \%$ in both cases).

6. While output count rates with optimum filters are limited to fewer than $5 \mathrm{cps}, 260 \mathrm{cps}$ are possible with the Pixie-16 for energy resolutions of $0.1 \%$ or better.

These results establish that a) the hardware architecture and b) the digital filter algorithms used for our HPGe pulse processing electronics, suitably modified, are capable of achieving energy resolutions with microcalorimeters comparable to the currently used LLNL electronics, which thus fulfills Objective 1 of the Phase I project. 


\section{Preliminary Hardware Design}

The Pixie-16 hardware used in the above measurements is a high speed spectrometer for HPGe detectors. The high speed components on the board are expensive; a single data acquisition (DAQ) channel costs $\sim \$ 1000$. For microcalorimeters, lower speed components can be used which are much cheaper. As we shall show, we are able to bring down the price to $\sim \$ 100 /$ channel in the design shown in Figure 18.

In this design, we propose to build a low cost, multi-channel " $\mu \mathrm{CAL}$ " spectrometer for use with microcalorimeter arrays. The design comprises a motherboard that contains a core digital processing design and a set of daughter cards that can be customized for different input signal types, e.g. individual analog inputs, analog signals multiplexed in time or frequency, or even digital inputs from systems already incorporating the digitization of the microcalorimeter signals into their detector electronics. By this means we can focus on the digital core processor and on optimizing its algorithms and performance and still produce an instrument that can be readily and economically adapted to whatever detector front ends the market or individual research produce by simply adding additional appropriate daughter cards.

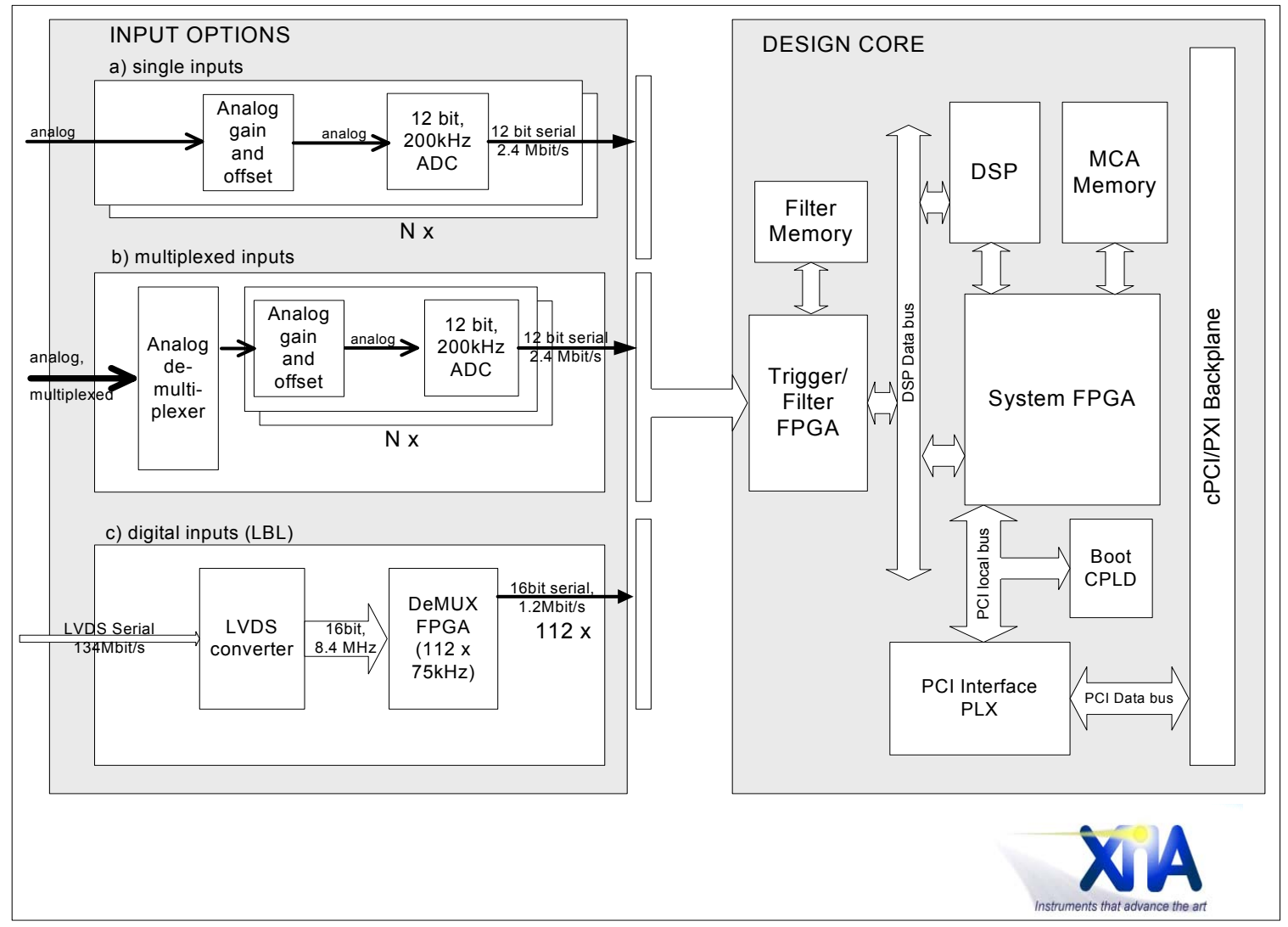

Figure 18: Architecture of low cost, multi-channel " $\mu$ CAL" spectrometer for microcalorimeters.

\subsection{Design of the $\mu \mathrm{CAL}$ 's Digital Core}

We plan to build the $\mu \mathrm{CAL}$ using the same CompactPCI/PXI form factor as the Pixie-16 used in Phase I measurements. CompactPCI is a rugged version of the commonly used PCI standard, defining industry standards for chassis, power supplies, and card modules. PXI is an extension of this standard that allows distribution of clocks and trigger signals over a chassis backplane. Modules in a CompactPCI/PXI chassis are linked with a PCI bridge to the PCI bus of a host desktop PC, where they appear as just another PCI board (e.g. a video card) plugged into the 
desktop. The PCI bridge allows high speed data readout from the module to the host PC; for modules developed by XIA we measured up to $80 \mathrm{Mbytes} / \mathrm{s}$ sustained in repeated block reads.

The core of the proposed $\mu$ CAL's digital processing will be a single high speed "trigger/filter" FPGA that will be capable of processing up to 112 channels in parallel. The digitized signal from each detector channel will arrive as a 12-16bit data stream at a rate of between 75 and $200 \mathrm{kHz}$, depending on the daughter card. The actual data connection to the FPGA does not affect production costs significantly and has been postponed to Phase II. For example, we might use a serial connection for each channel, requiring up to 224 input FPGA pins for ADC data or the data may come already time multiplexed from the daughter card, requiring far fewer pins. In addition, there may be 60-80 lines connecting to external filter memory, and up to 60 data, address and control lines to transfer the results to the DSP. All of these pin counts are well within the limits of large FPGA chips, e.g. those used on the Pixie-16. The FPGA itself will run at $80 \mathrm{MHz}$, i.e. $1000-400$ times faster than the ADCs. This is substantially slower than the FPGAs running on the existing Pixie-16s and will ease the e implementation by removing tight timing constraints. Since the FPGA operates so much faster, it can in each ADC sampling interval (5.0-13.3 $\mu \mathrm{s})$ process the filters of all 112 channels. Most of the processing consists of building the digital filter sums, which require the following main steps:

1. read filter sum from memory

2. add new ADC value to filter sum

3. read old ADC value from memory

4. subtract old ADC value from filter sum

5. store filter sum in memory

6. save $\mathrm{ADC}$ value in memory.

Even allowing for memory response times, these 6 steps can be executed in about 10 FPGA clock cycles when properly pipelined. In 1000-400 cycles, the same filter logic "circuit" in the FPGA can thus process 100-40 channels before the next ADC value comes in. Using several independent filter logic circuits and memories (for example, the FPGA in the Pixie-16 currently has 4 independent processing circuits), a single FPGA can thus process several hundred channels in parallel. Since the FPGA memory is limited, external memory chips will have to be used to store the filter sums, but these are not very expensive.

Just as in the Pixie-16, the trigger/filter FPGA detects pulses in the detector data streams and sends the raw filter sums for each event to the DSP for reconstruction of the pulse height. The DSP thus sees the combined triggered event rate of all channels, which, with a generous upper limit estimate of $1000 \mathrm{cps}$ x 100 channels, might approach $100 \mathrm{kcps}$ total. As the DSPs currently used on our high speed HPGe spectrometers can presently process $250 \mathrm{kcps}$ or more, using a single DSP will impose no limitation on throughput. Extending our existing spectrometer architecture, the pulse heights computed by the DSP are passed to a System FPGA for storage in an on-board MCA memory. The System FPGA also acts as an interface to the local PCI data bus and can distribute possible gate or trigger signals.

Unless all rejection cuts to eliminate "bad" events and all energy corrections for drifts etc can be applied in the DSP, it will be preferable to store for each additional list mode data for each processed pulse (including energy, time stamp, possibly rise time, initial slope and possibly other derived values) and then build MCA spectra on the host computer that has additional software to optimally apply appropriate cuts and corrections. For debugging purposes, it is will also be possible to store complete waveforms several milliseconds long. The list mode data can also be stored in a section of the MCA memory and then read out in a fast block transfer at the PCI transfer rate of $80 \mathrm{MByte} / \mathrm{s}$ or more. Arranging the list data into two 32bit words per event, the proposed MCA memory (4 chips with $256 \mathrm{~K} \times 32 \mathrm{bit}$ each) will be able to hold $512 \mathrm{~K}$ events, which will be read out in less than $0.1 \mathrm{~s}$. With 100 channels generating 1000 events/s each, the time to fill the memory will be about $5 \mathrm{~s}$, so that the readout dead time will be only $0.1 / 5=2 \%$. If it becomes desirable to eliminate even this tiny dead time, we can arrange the MCA memory for "ping-pong" transfers as we currently do in our high speed xMAP x-ray spectrometers.

Note that except for additional memory, the architecture of DSP, SystemFPGA, MCA memory and PCI interface is essentially equivalent to the architecture on the Pixie-16, which will allow us to reuse much of the existing firmware and DSP code. 


\subsection{Daughter Card Input Options}

\subsubsection{Single Analog Inputs}

In the simplest mode, the detector signals are directly and individually connected to the inputs of the $\mu \mathrm{CAL}$. Each detector channel would thus have its own analog stage, followed by an ADC. The analog stage will include offset adjustment, fixed gain, and a Nyquist filter, see Figure 19. The ADC will digitize the signal at a rate of 75$200 \mathrm{kHz}$ and send its (serial) data to the FPGA. On a 6U CompactPCI/PXI board similar to the Pixie-16, it will be possible to fit 32-64 channels of this kind. This circuit is nearly identical, except in bandwidth and sampling rate, to the one currently in use on many XIA processors. The novelty of the proposed design card will be the increased density (32-64 channels) and the plethora of serial connections to the trigger/filter FPGA.

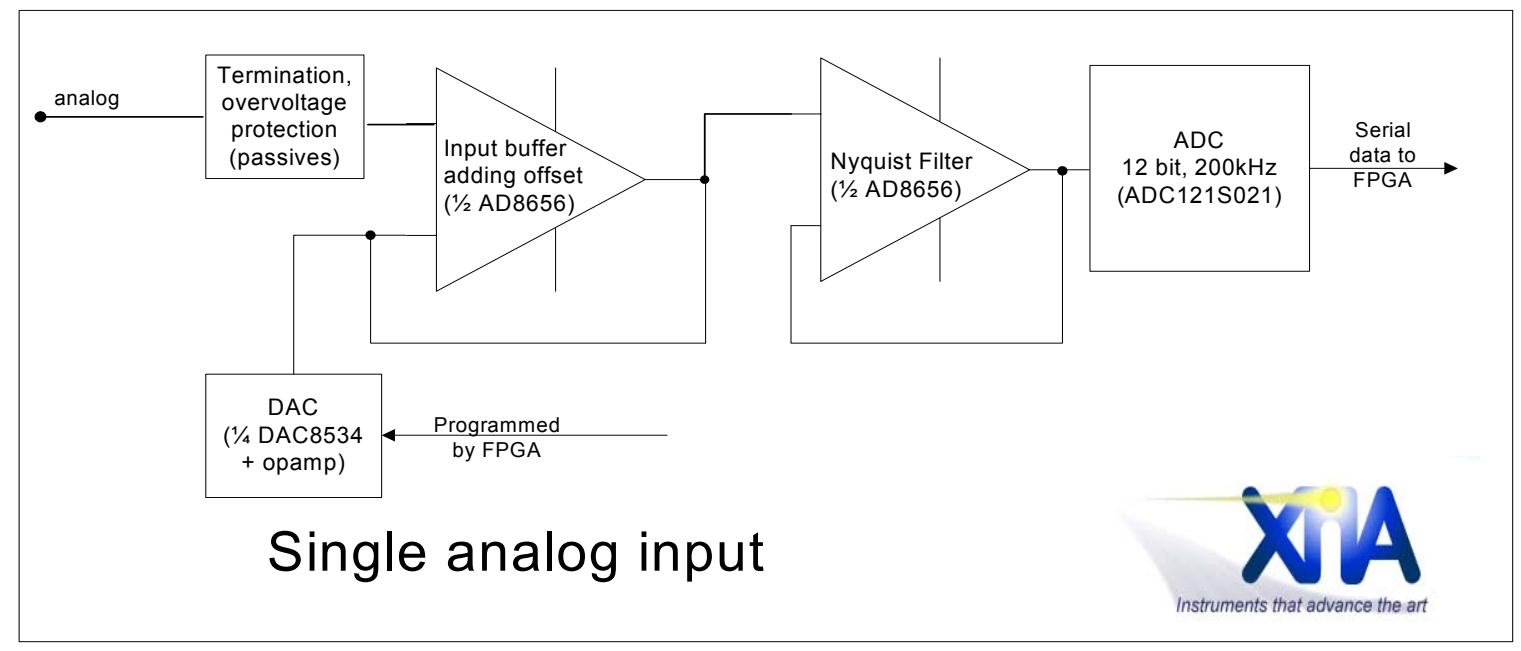

Figure 19: Architecture of an analog input channel.

\subsubsection{Multiplexed Analog Inputs}

Based on discussions with LLNL personnel, we expect that signals from multi-channel detectors will most likely come out of the detector multiplexed in either time or frequency (to limit the heat loss through the cables and reduce cabling complexity). For frequency multiplexing, we envisage two possible approaches. The first would be to add a matching commercially available de-multiplexing stage in front of the analog circuitry described above. The second would be to digitize the signals directly and then use an FPGA to de-multiplex them and present them to the trigger/filter FPGA as a time multiplexed series of digital values similar to the LBL output discussed below.

Time multiplexing relies on switching the output signal between different microcalorimeter channels at a rate of typically $1-2 \mu$ s between channel changes, for example: cycling through all 8 channel in a system in 8-16 $\mu$ s [14]. The multiplexer's output may take $\sim 0.5 \mu$ s to settle after a switch and can then be averaged for another $0.5 \mu$ s to obtain a sampling point for this channel. An input stage for receiving such a time multiplexed signal might then consist of a higher speed $\mathrm{ADC}(2-10 \mathrm{MHz})$ that continuously samples the multiplexed detector output at a high rate and passes them to an FPGA. The FPGA would also receive a digital input to synchronize the high speed digital data stream with the multiplexer switching between channels. Thus the FPGA can sort the data stream into different channels according to the synchronization signal, block data corresponding to the settling periods and average data over the valid periods to obtain one sampling point per channel per cycle.

The actual solution in either of these options will depend on the actual detector design and multiplexing method empolyed. We therefore do not go into further details for a generic design, but we instead consider a particular multiplexed solution in the following section.

\subsubsection{LBL Digital Outputs}

Several microcalorimeters currently in use or in planning use a readout and digitizing board as part of the detector electronics that is developed at the Physiscs Departments of UC Berkely (A. Lee) and LBL (H. Spieler). With an appropriate input daughterboard, our proposed hardware will also be able use the output from this board, which is a LVDS signal from 112 multiplexed channels digitized at 16 bit and $75 \mathrm{kHz}$ (i.e. a serial data stream of $134 \mathrm{Mbit} / \mathrm{s})$. The $\mu \mathrm{CAL}$ would first use a matching LVDS converter to recover the 16 bit data stream (at $8.4 \mathrm{MHz})$ 
and de-multiplex this data in an FPGA (either in a separate chip in the daughter card or directly in the trigger/filter FPGA).

\subsection{Hardware Bill of Materials and Pricing}

The estimated cost of the hardware components is shown below. Assuming a conservative 32 channels per board, the components for a single analog input daughterboard cost about $\$ 16.04$, the cost of the digital processing core per channel is about $\$ 10.67$. Pricing is accurate as of Feb/March 2006 and assumed purchase quantities $\sim 100$ for ICs and $\sim 1000$ for passives. The total price per channel is thus about $\$ 27$ plus cost of stuffing, testing, and a fraction of the PCB setup charges. In this context, it is worth noting that XIA typically applies a scaling factor of 68 in setting an instrument's sales price based on its parts cost, the difference being used to cover all the associated costs including production, engineering, sales, documentation, ongoing support, and some profit to invest in new product development. Thus, in this single analog daughterboard design, which would typically be employed for arrays with small numbers of detectors, the sales price/channel projected from this estimate would be about $\$ 160$. Further engineering to remove expensive parts, particularly the pricy low temperature coefficient resistors could lower the price further.

For the input option of LBL digital inputs, the digital data stream would only de-serialized on the input daughterboard. All further processing will take place in the core design. The daughterboard's parts cost per channel is then less than $\$ 1$ and the whole processor cost per channel is about $\$ 12$, for a projected instrument sales price of under $\$ 100$.

Overall, this demonstrates that a price of $\$ 100$ per channel is economically very feasible and thus fulfils Objective 2 of the Phase I project.

\begin{tabular}{|c|c|c|c|c|c|c|c|}
\hline Item & Description/Function & Part Number & Manufacturer & $\begin{array}{l}\text { Unit } \\
\text { Cost }\end{array}$ & $\begin{array}{l}\text { Qty per } \\
\text { board }\end{array}$ & $\begin{array}{c}\text { Total per } \\
\text { board }\end{array}$ & $\begin{array}{l}\text { Total per } \\
\text { channel }\end{array}$ \\
\hline 1 & $\mathrm{PCl}$ interface & PCI9054-AC50BI & PLX & 29.00 & 1 & 29.00 & 0.91 \\
\hline 2 & Boot CPLD & XC2C256-6TQ144C & Xilinx & 20.19 & 1 & 20.19 & 0.63 \\
\hline 3 & MCA memory, 256k x 32bit & CY7C1354C-200ACX & Cypress & 12.08 & 4 & 48.32 & 1.51 \\
\hline 4 & Filter memory & CY7C1354C-200ACX & Cypress & 12.08 & 2 & 24.16 & 0.76 \\
\hline 5 & System FPGA & XC3S200-4FT256C & Xilinx & 24.47 & 1 & 24.47 & 0.76 \\
\hline 6 & DSP & ADSP-2185MKST-300 & ADI & 19.80 & 1 & 19.80 & 0.62 \\
\hline 7 & Filter FPGA & XC3S1500-4FG456C & Xilinx & 74.58 & 1 & 74.58 & 2.33 \\
\hline 8 & passives & generic & generic & 0.03 & 300 & 9.00 & 0.28 \\
\hline 9 & power regulators & generic & generic & 5.00 & 5 & 25.00 & 0.78 \\
\hline 10 & $\mathrm{cPCl}$ connectors & $2 \mathrm{~mm}$ hard metric & generic & 6.00 & 2 & 12.00 & 0.38 \\
\hline 11 & high density connectors & generic & generic & 10.00 & 1 & 10.00 & 0.31 \\
\hline 12 & front panel & generic & generic & 15.00 & 1 & 15.00 & 0.47 \\
\hline \multirow[t]{2}{*}{13} & PCB board & PCB & generic & 30.00 & 1 & 30.00 & 0.94 \\
\hline & & & & & & 341.52 & 10.67 \\
\hline
\end{tabular}


Component Cost: Single Analog Input

Item Description/Function Part Number Manufacturer Unit Qty per Total per

Cost channel channel

\begin{tabular}{llllrrr}
\hline 1 & 12bit, 50-200kHz ADC ADC121S021 & National & 1.30 & 1 & 1.30 \\
2 & Buffer opamp & AD8656 & Analog Devices & 1.10 & 0.5 & 0.55 \\
3 & DAC opamp & AD8656 & Analog Devices & 1.10 & 0.5 & 0.55 \\
4 & Nyquist filter opamp & AD8656 & Analog Devices & 1.10 & 0.5 & 0.55 \\
5 & DAC & DAC8534 & Texas Instruments & 8.75 & 0.25 & 2.19 \\
6 & Passives & generic & generic & 0.03 & 30 & 0.90 \\
7 & Precision resistors & generic & generic & 0.25 & 20 & 5.00 \\
8 & PCB board & PCB & generic & 5.00 & 1 & 5.00 \\
\hline
\end{tabular}

Component Cost: LBL Digital Outputs

\begin{tabular}{|c|c|c|c|c|c|c|}
\hline Item & Description/Function & Part Number & Manufacturer & $\begin{array}{l}\text { Unit } \\
\text { Cost }\end{array}$ & $\begin{array}{l}\text { Qty per } \\
\text { channel }\end{array}$ & $\begin{array}{l}\text { Total per } \\
\text { channel }\end{array}$ \\
\hline 1 & LVDS converter & MAX9206 or similar & Maxim & 12.00 & 0.01 & 0.12 \\
\hline 2 & passives & generic & generic & 0.03 & 0.3 & 0.01 \\
\hline 1 & PCB board & PCB & generic & 5.00 & 0.01 & 0.05 \\
\hline
\end{tabular}

\section{Summary of Phase I Project}

In summary, in measurements with a microcalorimeter at LLNL, the adapted Pixie-16 spectrometer achieved an energy resolution of $0.062 \%$, significantly better than the targeted resolution of $0.1 \%$ in the Phase I proposal and easily matching resolutions obtained with LLNL readout electronics and optimum filtering (0.066\%). The theoretical maximum output count rate for the filter settings used to achieve this resolution is about $120 \mathrm{cps}$. If the filter is adjusted for maximum throughput with an energy resolution of $0.1 \%$ or better, rates of $260 \mathrm{cps}$ are possible. This is 20-50 times higher than the maximum count rates of about $5 \mathrm{cps}$ with optimum filters for this detector. While microcalorimeter measurements were limited to count rates of $\sim 1.3 \mathrm{cps}$ due to the strength of available sources, pulser measurements demonstrated that measured energy resolutions were independent of counting rate to output counting rates well in excess of 200cps or more. These results thus fulfill Objective 1 of the Phase I project.

We also developed a preliminary hardware design of a spectrometer module, consisting of a digital processing core and several input options that can be implemented on daughter boards. Depending upon the daughter board, the total parts cost per channel ranged between $\$ 12$ and $\$ 27$, resulting in projected product prices of $\$ 80$ to $\$ 160$ per channel. This demonstrates that a price of $\$ 100$ per channel is economically very feasible for large microcalorimeter arrays and thus fulfils Objective 2 of the Phase I project. 